\title{
Single Bursts of Individual Granule Cells Functionally Rearrange Feedforward Inhibition
}

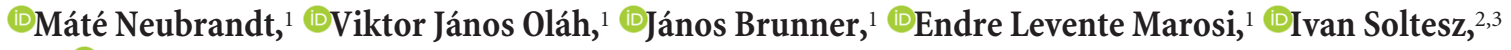 \\ and ${ }^{-J a ́ n o s ~ S z a b a d i c s ~}{ }^{1}$ \\ ${ }^{1}$ Institute of Experimental Medicine, Hungarian Academy of Sciences, Budapest, 1083, Hungary, ${ }^{2}$ Department of Neurosurgery, and ${ }^{3}$ Stanford \\ Neurosciences Institute, Stanford University, Stanford, California 94305
}

\begin{abstract}
The sparse single-spike activity of dentate gyrus granule cells (DG GCs) is punctuated by occasional brief bursts of 3-7 action potentials. It is well-known that such presynaptic bursts in individual mossy fibers (MFs; axons of granule cells) are often able to discharge postsynaptic CA3 pyramidal cells due to powerful short-term facilitation. However, what happens in the CA3 network after the passage of a brief MF burst, before the arrival of the next burst or solitary spike, is not understood. Because MFs innervate significantly more CA3 interneurons than pyramidal cells, we focused on unitary MF responses in identified interneurons in the seconds-long postburst period, using paired recordings in rat hippocampal slices. Single bursts as short as 5 spikes in $<30 \mathrm{~ms}$ in individual presynaptic MFs caused a sustained, large increase (tripling) in the amplitude of the unitary MF-EPSCs for several seconds in ivy, axo-axonic/chandelier and basket interneurons. The postburst unitary MF-EPSCs in these feedforward interneurons reached amplitudes that were even larger than the MF-EPSCs during the bursts in the same cells. In contrast, no comparable postburst enhancement of MF-EPSCs could be observed in pyramidal cells or nonfeedforward interneurons. The robust postburst increase in MF-EPSCs in feedforward interneurons was associated with significant shortening of the unitary synaptic delay and large downstream increases in disynaptic IPSCs in pyramidal cells. These results reveal a new cell type-specific plasticity that enables even solitary brief bursts in single GCs to powerfully enhance inhibition at the DG-CA3 interface in the seconds-long time-scales of interburst intervals.
\end{abstract}

Key words: burst firing; feedforward inhibition; hippocampus

\section{Significance Statement}

The hippocampal formation is a brain region that plays key roles in spatial navigation and learning and memory. The first stage of information processing occurs in the dentate gyrus, where principal cells are remarkably quiet, discharging low-frequency single action potentials interspersed with occasional brief bursts of spikes. Such bursts, in particular, have attracted a lot of attention because they appear to be critical for efficient coding, storage, and recall of information. We show that single bursts of a few spikes in individual granule cells result in seconds-long potentiation of excitatory inputs to downstream interneurons. Thus, while it has been known that bursts powerfully discharge ("detonate") hippocampal excitatory cells, this study clarifies that they also regulate inhibition during the interburst intervals.

\section{Introduction}

Bursts of high-frequency action potentials (APs) are distinct neuronal signals generated by nonlinear dendritic processes in response to coincident inputs that result in a reliably transmitted

\footnotetext{
Received June 8, 2017; revised Dec. 7, 2017; accepted Jan. 5, 2018.

Author contributions: M.N., I.S., and J.S. designed research; M.N., V.J.O., J.B., and E.M. performed research;M.N. and J.S. analyzed data; I.S. and J.S. wrote the paper.

This work was supported by Wellcome Trust International Senior Research Fellowship 087497 to J.S., Hungarian Academy of Sciences Lendület Initiative LP-2009-009 to J.S., Hungarian Brain Research Program KTIA_13_NAP-A-I/5 to J.S., Stephen W. Kuffler Research Foundation to V.J.O., and National Institutes of Health Grant NS35915 to I.S. We thank Dr. Thomas Südhof for helpful discussions; Drs. Mark Eyre and Katalin Tóth for comments and suggestions on a previous version of the manuscript; and Dóra Kókay and the late Andrea Juszel for technical assistance. M.N., V.J.0., and J.B. were students at the János Szentágothai Doctoral School of Neurosciences, Semmelweis University.

The authors declare no competing financial interests.
}

output (Lisman, 1997; Harris et al., 2001; Xu et al., 2012; Apostolides et al., 2016). The granule cells of the dentate gyrus (DG GCs) are among the most quiescent neurons in the cortical mantle with an unusually low overall firing rate $(<1 \mathrm{~Hz}$ in vivo), but they are also capable of discharging brief bursts of 3-7 APs within tens of milliseconds $(100-200 \mathrm{~Hz})$, separated by long silent periods (2-15 s). These bursts occur during distinct behaviors (Buz-

Correspondence should be addressed to Dr. János Szabadics, Institute of Experimental Medicine, Hungarian Academy of Sciences, Szigony utca 43, Budapest, 1083, Hungary. E-mail: szabadics.janos@koki.mta.hu.

DOI:10.1523/JNEUROSCI.1595-17.2018

Copyright $\odot 2018$ Neubrandt et al.

This is an open-access article distributed under the terms of the Creative Commons Attribution License Creative Commons Attribution 4.0 International, which permits unrestricted use, distribution and reproduction in any medium provided that the original work is properly attributed. 
sáki et al., 1983; Jung and McNaughton, 1993; Henze et al., 2002; Pernía-Andrade and Jonas, 2014; Diamantaki et al., 2016; Danielson et al., 2017; GoodSmith et al., 2017; Senzai and Buzsáki, 2017) and are channeled to the CA3 region by the axons of the DG GCs, the mossy fibers (MF), where each MF innervates only 11-18 CA3 pyramidal cells but several times more GABAergic cells (40-50 cells) (Acsády et al., 1998).

The well-studied response of postsynaptic CA3 pyramidal cells during MF bursts is characterized by strong short-term facilitation that enables the MF-generated EPSPs to reach firing threshold and almost invariably discharge the pyramidal cells, a property referred to as "conditional detonation" (conditional in the sense that if there is a presynaptic burst, then there is pyramidal cell firing) (Salin et al., 1996; Tóth et al., 2000; Gundlfinger et al., 2010; Chamberland et al., 2014; Vyleta et al., 2016). The MFs innervate at least four distinct types of GABAergic interneurons in the CA3, with the MF-interneuron synaptic connections displaying cell type-specific properties and short-term plasticity rules that result in a weaker excitation of the MF-CA3 GABAergic interface than the conditional detonation displayed by the CA3 pyramidal cells (Henze et al., 2002; Lawrence and McBain, 2003; Mori et al., 2004; Nicoll and Schmitz, 2005; Szabadics and Soltesz, 2009; Zucca et al., 2017). In contrast to the availability of cell type-specific information about the postsynaptic events triggered by MF activity during the burst in CA3 pyramidal cells and distinct interneuron types, virtually nothing is known about how physiologically realistic (i.e., brief, single) bursts of APs in individual MFs shape the MF-CA3 GABAergic network in the seconds-long interburst period, after the occurrence of one burst and before the arrival of the next. Indeed, while previous studies have shown that changes in the MF-evoked excitation of CA3 inhibitory cells can persist for tens of seconds or even minutes after prolonged high-frequency stimulation (300 APs at 30-40 Hz) (Alle et al., 2001; Mori et al., 2007), the effect of shorter physiological MF burst activity on CA3 interneurons remains unknown.

Here we combined paired recordings from the presynaptic GCs that reside in the CA3, or directly from MF boutons of DG GCs, with recordings from postsynaptic, post hoc identified CA3 cells to study how single, brief bursts of APs in individual MFs modify the unitary MF responses in the postburst period. The results show that even single bursts formed by a few spikes occurring in $<30 \mathrm{~ms}$, representing physiologically relevant bursts that actually occur in vivo, cause a sustained (up to $8 \mathrm{~s}$ ) tripling of the amplitude of the unitary MF-EPSCs in feedforward interneurons (FF-INs), including axo-axonic/chandelier cells, two types of basket cells and ivy cells. The tripling of the MF-EPSCs after a single brief burst activity was specific to FF-INs, and no comparable postburst potentiation could be observed in either CA3 pyramidal cells or extrahippocampally projecting GABAergic neurons. Furthermore, the postburst enhancement of the MFEPSCs in FF-INs was strong enough to cause large, sustained increases in disynaptic IPSCs in randomly sampled CA3 pyramidal cells.

These results reveal a novel type of MF plasticity that makes it possible for even solitary, brief bursts of APs in single GCs to enhance CA3 inhibition at time-scales that are commensurate with the physiological interburst intervals.

\section{Materials and Methods}

Slice preparation, recording conditions, and processing for anatomy. For acute slice preparations, adolescent Wistar rats (postnatal day 21-45, both sexes) were deeply anesthetized with isoflurane (in accordance with the ethical guidelines of the Institute of Experimental Medicine Protec- tion of Research Subjects Committee; 22.1/1760/003/2009), and $350 \mu \mathrm{m}$ slices were cut in ice-cold ACSF containing the following (in mM): 85 $\mathrm{NaCl}, 75$ sucrose, $2.5 \mathrm{KCl}, 25$ glucose, $1.25 \mathrm{NaH}_{2} \mathrm{PO}_{4}, 4 \mathrm{MgCl}_{2}, 0.5 \mathrm{CaCl}_{2}$, and $24 \mathrm{NaHCO}_{3}$. The slices were cut from the dorsal and medial part of the hippocampus using a standard vibratome (Leica VT1200S, 0.08 $\mathrm{mm} / \mathrm{s}$ speed and $1 \mathrm{~mm}$ amplitude). The slice orientation was, as in Szabadics and Soltesz (2009), intended to be parallel to the MFs and many CA3 dendrites to maximize the preservation of MF connections to the CA3. The slices were incubated at $32^{\circ} \mathrm{C}$ for $60 \mathrm{~min}$ after sectioning and were then stored at room temperature until they were used for recordings within $10 \mathrm{~h}$. The cells were visualized with an upright microscope (Eclipse FN-1; Nikon) with infrared $(900 \mathrm{~nm})$ Nomarksi differential interference contrast optics ( $40 \times 0.8 \mathrm{NA}$ water-immersion objective). The standard recording ACSF was composed of the following (in $\mathrm{mm}$ ): $126 \mathrm{NaCl}, 2.5 \mathrm{KCl}, 26 \mathrm{NaHCO}_{3}, 2 \mathrm{CaCl}_{2}$ (unless stated otherwise), $2 \mathrm{MgCl}_{2}, 1.25 \mathrm{NaH}_{2} \mathrm{PO}_{4}$, and 10 glucose at $35 \pm 0.5^{\circ} \mathrm{C}$.

Electrophysiological recordings were obtained with MultiClamp 700B amplifiers (Molecular Devices) and pClamp10 software (sampling rate $50 \mathrm{kHz}$ ). Postsynaptic cells were voltage-clamped at $-70 \mathrm{mV}$ (low-pass filtered at $4-6 \mathrm{kHz})$. Series resistance $(5-30 \mathrm{M} \Omega$ ) was monitored by the capacitive artifact in response to a $5 \mathrm{mV}$ step in each trace. Presynaptic CA3 GCs were recorded in current clamp (low-pass filtered at $10-20 \mathrm{kHz}$ ), whereas large MF terminals were preferentially assessed in cell-attached configuration with pipettes containing intracellular solution (Szabadics and Soltesz, 2009; Vyleta and Jonas, 2014); some giant MF terminals were recorded in current-clamp mode. The pipette capacitance was greatly reduced, but not fully neutralized, in the bridge-balance-compensated current-clamp recordings. Recording pipettes were pulled from either thin- or thick-walled ( 1.12 or $0.86 \mathrm{~mm}$ inner diameter, 1.5 outer diameter) borosilicate glass capillaries; the pipette resistance ranged between 3 and $4.5 \mathrm{M} \Omega$ for the somatic recordings or between 10 and $12 \mathrm{M} \Omega$ for the axonal recordings.

Three different intracellular solutions were used. Under standard recording conditions, pairs were recorded in an intracellular solution containing the following (in mM): $90 \mathrm{~K}$-gluconate, $43.5 \mathrm{KCl}, 1.8 \mathrm{NaCl}, 1.7 \mathrm{MgCl}_{2}, 0.05$ EGTA, 10 HEPES, $2 \mathrm{Mg}$-ATP, $0.4 \mathrm{Na}_{2}$-GTP, 10 phosphocreatine-disodium, and 8 biocytin ( $\mathrm{pH} 7.25$ ). In one set of experiments (see Fig. $3 B-D$ ), presynaptic recordings were performed with a modified version of the first intracellular solution ( $40 \mathrm{~mm} \mathrm{KCl}$ was substituted for $\mathrm{CsCl}$ ) to increase the release probability of the MF-spiny lucidum cell (SLC) connections. The third intracellular solution was optimal for assessing the disynaptic connections because of the large difference between the reversal potentials of the GABAergic and glutamatergic events; specifically, the postsynaptic pyramidal cells were patched with a low $\left[\mathrm{Cl}^{-}\right]$intracellular solution composed of the following (in mM): $133.5 \mathrm{~K}$-gluconate, $1.8 \mathrm{NaCl}, 1.7 \mathrm{MgCl}_{2}, 0.05 \mathrm{EGTA}, 10 \mathrm{HEPES}, 2$ Mg-ATP, $0.4 \mathrm{Na}_{2}$-GTP, 10 phosphocreatine-disodium, and 8 biocytin ( $\mathrm{pH}$ 7.25). The chemicals for the intracellular and extracellular solutions were purchased from Sigma-Aldrich, and the various pharmacons were purchased from Tocris Bioscience. DCG IV $(1 \mu \mathrm{M})$, MSOP $(150 \mu \mathrm{M})$, and SR 95531 (gabazine, $5 \mu \mathrm{M}$ ) were dissolved in ACSF. Phorbol 12,13-dibutyrate (PDBu, $1 \mu \mathrm{M})$, Go 6976 (250 nM), GF 109203X (1 $\mu \mathrm{M})$, calphostin C (1 $\mu \mathrm{M})$, U $73122(2.5 \mu \mathrm{M})$, BINA (5 $\mu \mathrm{M})$, AMN $082(1 \mu \mathrm{M})$, and KT5720 (200 nM) were dissolved first in DMSO and diluted at least 2000 times in ACSF. EGTA (0.5-2.5 mM), PKC19-36 (100 $\mu \mathrm{M})$, and PKA inhibitory fragment 6-22 amide (PKI, $2.5 \mu \mathrm{M}$ ) were added to the intracellular solution. In the case of GF109203X, Go6976, and KT5720, the slices were also preincubated before recordings. Otherwise, the drugs were added to the perfused ACSF.

After the recordings, the slices were fixed for $1 \mathrm{~d}$ in $0.1 \mathrm{M}$ phosphate buffer containing $2 \%$ PFA and $0.1 \%$ picric acid at $4^{\circ} \mathrm{C}$. After fixation, the slices were resectioned at $60 \mu \mathrm{m}$. For immunocytochemistry, the sections were incubated with one or two of the following primary antibodies against parvalbumin (PV; PV25 and PV27, 1:1000, polyclonal rabbit, Swant), SATB1 (sc-5989, 1:400, polyclonal goat, Santa Cruz Biotechnology), cholecystokinin (CCK; C2581, 1:1000, polyclonal rabbit, Sigma-Aldrich), somatostatin (MAB354, 1:500, monoclonal rat, Millipore Bioscience Research Reagents), or neuronal nitric oxide synthase (N2280, 1:500 mouse, Sigma-Aldrich) overnight in $0.5 \%$ Triton X-100 and $2 \%$ normal goat serum or horse serum containing TBS buffer at $4^{\circ} \mathrm{C}$. The immunoreactions were visualized with AlexaFluor-488- or AlexaFluor-594-conjugated secondary goat or donkey antibodies (1:500; Invitrogen) against rabbit, goat, mouse, 
and rat IgGs, and biocytin staining was revealed using AlexaFluor-350- or AlexaFluor-488-conjugated streptavidin. The recorded cells were analyzed on epifluorescence microscope (DM2500, Leica). Multiple image stacks were acquired from a $60-\mu \mathrm{m}$-thick slice to visualize the axonal and dendritic arbors. The maximum-intensity-projected black-and-white fluorescence images were inverted for better visualization of a large part of the dendritic and axonal arborization. One recorded pair (Fig. 3-1, available at https://doi. org/10.1523/JNEUROSCI.1595-17.2018) and a few other test samples were processed for DAB staining (Szabadics and Soltesz, 2009). Because DAB staining did not provide a better substrate for anatomical identification than fluorescent labeling, the other analyses were completed using the latter, methodologically simpler staining method.

Cell types. A total of 223 monosynaptic pairs were included in this work. However, the majority of the analysis included only those pairs where the postsynaptic cell was identified as an FF-IN ( $n=116$ pairs, including IvyC, AAC, PV + BC, and CCK + IN types), SLC ( $n=25$ pairs), or pyramidal cell ( $n=12$ pairs). The single MF source for monosynaptic connections was either a giant MF terminal in the stratum lucidum $(n=$ $7)$ or a CA3 GC $(n=216$, including divergent connections from the same presynaptic source). The giant MF terminals specifically innervate pyramidal cells, whereas GABAergic cells receive MF inputs from small en passant or filopodial boutons. Thus, giant MF terminal recordings in whole-cell mode are able to maintain stable release onto GABAergic cells (Szabadics and Soltesz, 2009). The other presynaptic MF source was CA3 GCs, which provide a reliable, easily accessible, and stable model for studying single MF inputs using standard whole-cell paired recordings (Szabadics et al., 2010). CA3 GCs were identified based on the location of their soma, typical GC firing pattern, and polarized morphology; straight spiny dendrites were mostly oriented toward the stratum lacunosummoleculare, and the axons with large MF terminals were in stratum lucidum or stratum pyramidale. The CA3 GCs within this sample showed similar cellular properties as fully mature GCs (Brunner et al., 2014).

Postsynaptic cells were classified based on multiple criteria. IvyCs $(n=$ 87 pairs) were identified by their characteristic firing pattern (late firing, large and slow after hyperpolarization), short dendrites, and dense axon arborization mainly in the strata radiatum and oriens. Nine of the 14 tested IvyCs were neuronal nitric oxide synthase-positive, and none of the tested IvyCs was positive for CCK or somatostatin $(n=20$ and $n=$ 20 , respectively). PV $+\mathrm{BCs}(n=5)$ were identified based on their fastspiking activity and axons that specifically targeted the stratum pyramidale. All tested PV + BCs were immunopositive for PV $(n=4)$ and SATB1 $(n=2)$ (Viney et al., 2013). AACs $(n=10)$ were identified based on their fast spiking activity and characteristic axons apparently outlining the axon initial segments at the border of strata pyramidale and oriens, as well as the presence of PV ( 8 of the 9 tested cells) and lack of SATB1 immunopositivity $(n=6)$. Regular-spiking and CCK-expressing neurons $(n=11)$ were classified as CCK + INs, including both basket cells and dendrite targeting cells. SLCs $(n=25)$ were identified based on their densely spiny dendrites, which were usually restricted to the strata lucidum and pyramidale and somatostatin immunopositivity (15 positive of 19 tested) and lack of CCK expression ( $n=13$ tested). Pyramidal cells were targeted in the stratum pyramidale and were identified based on their typical firing pattern and morphology, including thick, densely spiny dendrites and complex spines in the stratum lucidum.

Disynaptic IPSCs (diIPSCs) were assessed in paired recordings of MF terminals or CA3 GCs and pyramidal cells in a separate set of experiments using less $\mathrm{Cl}^{-}$-containing intracellular solution for the unequivocal distinction of EPSCs and IPSCs. The membrane potential was held usually at $-50 \mathrm{mV}$ (above the reversal potential of $\mathrm{Cl}^{-}$). diEPSCs were observed in postsynaptic interneurons. The onset delays of the diEPSCs were clearly distinguishable from monosynaptic EPSCs (see Fig. 7E). The excitatory nature of the events was confirmed by depolarizing the postsynaptic cells close to the reversal potential of $\mathrm{Cl}^{-}$or by gabazine wash in. diIPSCs and diEPSCs were analyzed in a predefined time window (1.56.5 and 2-7 ms, respectively, from the peak of the presynaptic AP), in which all events were counted, potentially including a few spontaneous events originating from other presynaptic sources.

Experimental design and statistical analysis. The postsynaptic responses were tested in each recorded pair with multiple (usually 3 or 4 ) different protocols, varying either the delay between the burst and the test pulses (between 0.1 and $13.5 \mathrm{~s}$ ) or the number and pattern of APs within the burst (1-20 APs), and the measurements were considered to be independent data points for the analysis. Each trace included one control, one burst, and one test stimuli. Control stimuli (usually $3 \mathrm{APs}$ at $20 \mathrm{~Hz}$ ) were followed by a single burst within $200 \mathrm{~ms}$. For investigating the temporal profile of the effects of single presynaptic bursts, the only difference between consecutive traces was the gap between the burst and test stimuli. The next trace was recorded after allowing for the responses to recover to control levels (at least $60 \mathrm{~s}$ ). For investigating the effects of various presynaptic burst patterns, we followed the same strategy as above, except the timing between the burst and test pulses were constant in individual protocols and only the pattern was varied (i.e., number or frequency of APs within the burst). After testing each different protocol once, we repeated them consecutively to collect the sufficient numbers of traces. Thus, the different protocols gave directly comparable results and included reliable internal controls (the control responses before burst). Only those consecutive traces were considered for analysis in which the amplitudes, short-term plasticity and kinetics of the control responses were stable (i.e., without run down or long-term changes).

EPSC peak amplitudes were measured relative to a preceding baseline period on average traces. Minimum 3, but usually 5-10 traces were averaged for every protocol. For control responses (i.e., before bursts), all traces were averaged, thus, the same control was considered for 3-4 different postburst data points within each pair. Paired pulse ratios (PPRs) were calculated from the average traces by dividing the average of the second and the third EPSC amplitudes with the first EPSC amplitude of the $20 \mathrm{~Hz}$ control and test responses. Synaptic delays were measured at individual events from the peak of the presynaptic AP to the onset of the events. Data are presented as mean \pm SE. Statistical tests are indicated within the text. Normality of distributions was tested with the ShapiroWilks test. One-sample or two-sample unpaired Student's $t$ test and paired Student's $t$ test are indicated as $t$ test and paired $t$ test within the text. Degrees of freedom are indicated after each $t$ (for $t$ tests) or $F$ (for ANOVA) values in parentheses.

\section{Results}

Paired recordings to test the effects of physiologically relevant burst activity in single GCs on identified postsynaptic

\section{CA3 cells}

We tested the postburst consequences of single MF bursts in various types of postsynaptic CA3 neurons using unitary responses in paired patch-clamp recordings (for the experimental arrangement, see Fig. $1 A$, left). For the presynaptic side, we obtained precise control of the synaptic outputs of single presynaptic MFs by using two different approaches. First, direct intracellular or cell-attached recordings from MF terminals allowed us to assess the properties of unitary EPSCs from individual DG GCs to CA3 neurons (Szabadics and Soltesz, 2009). Second, somatic recordings from ectopic GCs (CA3 GCs), whose synaptic properties are indistinguishable from those of DG GCs (Szabadics et al., 2010), allowed for a reliable comparison of MF-to-CA3 neuron EPSCs across multiple protocols, which require long-term recordings. The results from the two types of presynaptic MF recordings were comparable and were therefore grouped together for analysis (for direct comparison of the effects of the two MF sources, see below). The postburst potentiation was primarily assessed by comparing the relative amplitudes of preburst control and postburst test MF-EPSCs (e.g.; Fig. 1B, $y$ axis) with the test responses following the bursts within hundreds of milliseconds to several seconds $(x$ axis in Fig. $1 B)$.

In total, we tested the effects of single bursts (in our standard protocol with $15 \mathrm{AP}$ at $150 \mathrm{~Hz}$ ) in 78 connected pairs in which the postsynaptic interneurons were identified as belonging to the previously reported feedforward category (FF-IN), including ivy cells (IvyCs, $n=55$ pairs), axo-axonic cells (AACs, $n=10$ pairs), $\mathrm{PV}$-expressing, fast-spiking basket cells ( $\mathrm{PV}+\mathrm{BCs}, n=5$ pairs), 
A
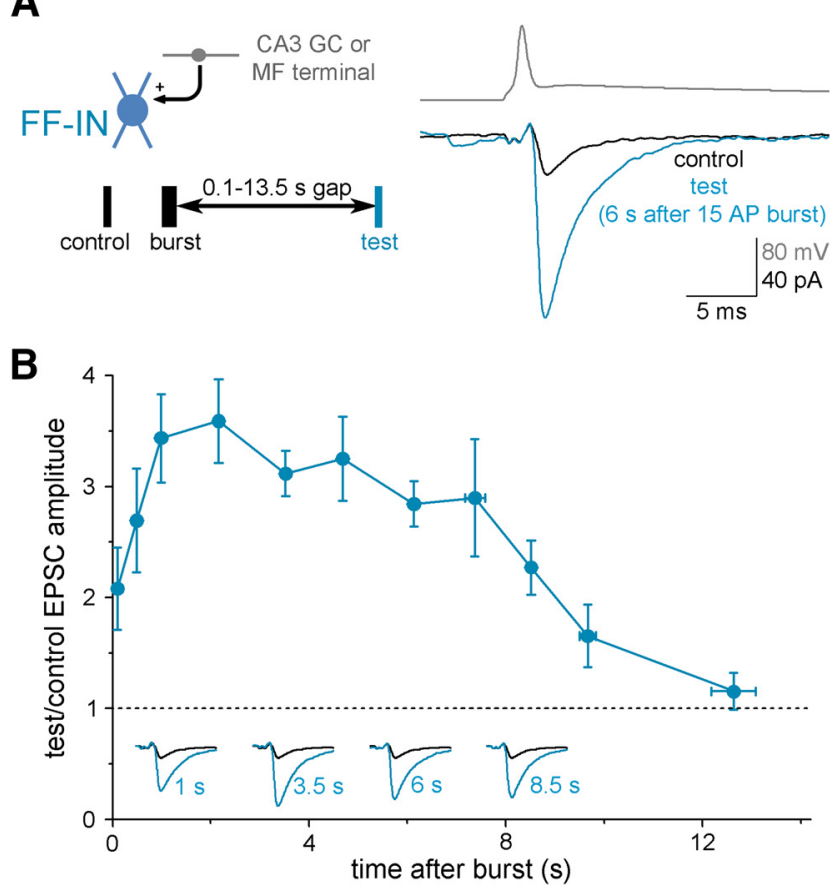

C
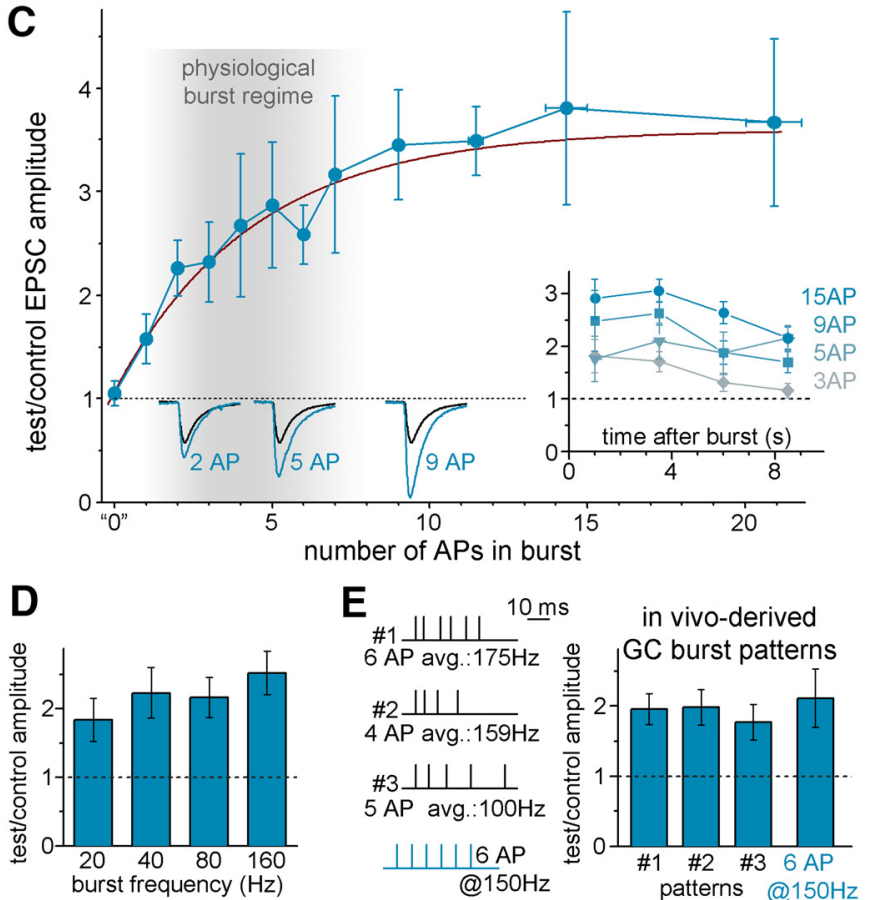

E

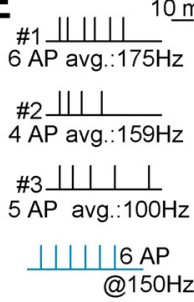

in vivo-derived

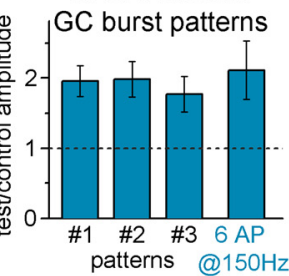

Figure 1. Single, brief MF bursts potentiate monosynaptic MF-EPSCs in CA3 FF-INs for several seconds. $A$, Schematic representation of the recording configuration for unitary MF responses in CA3 neurons. Presynaptic patch-clamp recording was obtained from a single giant MF terminal (most of these originate from DG GCs; Fig. 1-1, available at https://doi.org/10.1523/JNEUROSCI.1595-17. 2018) or a single CA3 GC (somatic recording; these cells are rarer but easier to record than the MF terminals); the postsynaptic cell was an FF-IN. MF output was first measured with a control stimulus, followed by a single 2-20 AP burst ( $150 \mathrm{~Hz})$; after various time delays $(0.1-13.5 \mathrm{~s})$, which approximated the typical physiological inactivity periods in $\mathrm{GC}$ in vivo, a test response was evoked. Right, Example traces of single-AP-evoked control and test EPSCs in an identified FF-IN (the illustrated recordings were from an IvyC; for the anatomy of the presynaptic and postsynaptic cells, see Fig. 1-1, available at https://doi.org/10.1523/JNEUROSCI.1595-17.2018). In most cases, control and test pulses contained 3 APs at 20 Hz to gain insight into possible changes in short-term plasticity after the bursts; plots in this and subsequent figures show responses to the first APs in the triplet, except in Figure 4C, D. B, Time course of the relative amplification of monosynaptic MF-EPSCs after single 15 AP presynaptic bursts in the same individual MF. Relative postburst EPSC amplitudes are shown (i.e., control relative amplitudes are 1, dashed line). The $x$ axis indicates the time of the test pulse after the burst. The graph includes all data points ( $n=280$, first APs) from identified FF-IN pairs ( $n=78$ pairs; including IvyCs: $n=55$ pairs; AACs: $n=10 ;$ PV +BC: $n=5 ; C C K+I N, n=8 ;$ for separate analysis of the burst-induced amplification in different postsynaptic cell types, see Figs. 1-1, 1-2, 1-3, and 1-4, available at https://doi.org/10.1523/JNEUROSCI.1595-17.2018). Insets, Example test responses in blue at different postburst delays; same postsynaptic lvyC as in $\boldsymbol{A}$; control traces are black. $\boldsymbol{C}$, Dependence of the postburst potentiation on AP numbers within the burst (from $n=99$ data points from $n=28$ pairs). Brown curve indicates the exponential fit of the data $\left(R^{2}=0.966\right)$. Gray area represents the typical range of GC bursts in vivo. Insets, Example test and control traces and the time course of the changes of the responses after 3,5,9, and $15 \mathrm{AP}$ bursts. $D$, Effects of single presynaptic bursts consisting of $8 \mathrm{APs}$ at 20, 40,80 , and $150 \mathrm{~Hz}$, on the same MF-FF-IN pairs ( $n=$ 7). $\boldsymbol{E}$, Effects of single bursts, whose patterns were obtained from in vivo recorded identified GCs: \#1, 6 APs with 3.7, 7.5, 4.54, 6.98, and 5.88 ms interspike intervals (Henze et al., 2002); \#2, 4 APs with 4, 6, and 9 ms interspike intervals (Pernía-Andrade and Jonas, 2014); \#3, 5 APs with 6, 8, 11, and 15 ms interspike intervals (Diamantaki et al., 2016 ); for comparison, the effect of a 6 AP 150 $\mathrm{Hz}$ burst is also shown. The effects were measured with each realistic burst protocol in the same pairs ( $n=8$ pairs, $3.6 \mathrm{~s}$ after burst).

and regular-spiking CCK-expressing interneurons (CCK+INs, $n=8$ pairs, including $n=3$ unequivocally identified CCK basket cells). The differences in the numbers of tested pairs reflect neither the occurrence of the cell types nor the probability of connectivity from GCs (Szabadics and Soltesz, 2009). The basal properties of MF-EPSCs onto FF-INs and their short-term plasticity, ranging between slight facilitation and slight depression (see below), were consistent with previous results (Tóth and McBain, 1998; Tóth et al., 2000; Szabadics and Soltesz, 2009; Torborg et al., 2010). The postsynaptic cells were recorded in voltage-clamp mode, minimizing confounds from spiking activity of the recorded postsynaptic cells to the measured changes.

\section{Seconds-long potentiation of MF-EPSCs in FF-INs after single MF bursts}

First, we studied the postburst consequences of single MF bursts consisting of 15 APs in $100 \mathrm{~ms}(150 \mathrm{~Hz})$. Such presynaptic AP bursts had surprisingly large effects on the MF-EPSCs in FF-INs, resulting in a tripling of the amplitudes of the test compared with the control responses for several seconds after the burst (Fig. 1A,B; Figs. 1-1, 1-2, 1-3, and 1-4, available at https://doi.org/10.1523/JNEUROSCI.1595-17.2018). Specifi- cally, the effect of the bursts in each pair ( $n=78$ pairs) was examined at 3 or 4 different postburst times for the test responses, and the average increase in test over control responses was $3.09 \pm$ 0.13 -fold between 1.5 and $6.7 \mathrm{~s}$ after the burst (control response: $-43.8 \pm 4.6 \mathrm{pA}$; test: $-109 \pm 8.6 \mathrm{pA} ; p=5 \times 10^{-36}, t_{(153)}=$ 16.58 , paired $t$ test, total number of postburst test responses at various postburst times, $n=154$; Fig. 1-1, available at https://doi.org/10.1523/JNEUROSCI.1595-17.2018).

As the plot in Figure $1 B$ indicates, the burst-induced increase in the MF-EPSCs in FF-INs was not instantaneous, and it gradually developed during the first second after presynaptic burst. After this initial increase, the burst-induced MF-EPSCs remained at a similarly enhanced level for about $8 \mathrm{~s}$ (comparison of the responses between 0.8 and $8 \mathrm{~s}$ after the burst, in 6 bins by one-way ANOVA: $\left.p=0.54, F_{(5,198,203)}=0.814\right)$, before the MF-EPSC amplitudes returned to baseline, control values $\left(p=0.081, t_{(5)}=\right.$ 2.184, $n=6$ timings, $t$ test, 9.5-13.5 s after burst). It should be noted that the postburst potentiation shown in Figure $1 B$ is distinct from the classical post-tetanic potentiation (Alle et al., 2001; Mori et al., 2007) not only because the latter typically requires considerably more intense presynaptic activity than our physiologically inspired burst protocol, but also because of its temporal 
profile since post-tetanic potentiation is largest immediately after the high-frequency stimulation and then continuously decays back to baseline. Interestingly, despite the specialized roles and synaptic-cellular mechanisms of the various interneurons within hippocampal circuits (Somogyi et al., 2014), the MF bursts had similar effects on all the postsynaptic FF-IN groups that we examined, including IvyCs, AACs, CCK + INs, and PV+BCs (relative increase in test/control responses: $3.15 \pm 0.16,2.88 \pm 0.32$, $2.98 \pm 0.64$, and $2.99 \pm 0.3$-fold amplification, respectively, oneway ANOVA, $p=0.91, F_{(3,150,153)}=0.187$; Figs. 1-1, 1-2, 1-3, $1-4)$.

\section{Robust effects of single bursts consisting of only a few} (3-5) APs

Although the results above were obtained using bursts in which the firing frequency represented typical GC burst (Henze et al., 2002; Pernía-Andrade and Jonas, 2014; GoodSmith et al., 2017) and these bursts contained considerably fewer APs than those in previously used paradigms (Alle et al., 2001; Mori et al., 2007), the 15 APs per burst that we used in the experiments described above were still more than what is found in typical GC bursts in vivo (2-7 APs) (Henze et al., 2002; Pernía-Andrade and Jonas, 2014). Therefore, we tested the effectiveness of shorter, truly physiological single bursts, aiming to determine the minimal presynaptic activity range that still results in postburst potentiation of MFEPSCs. In these experiments, the postburst timing of the test MF-EPSC was kept constant ( $3 \mathrm{~s}$ ) and the AP number within the burst was systematically varied between 1 and 20 spikes (e.g., effects of 2-5-9 or 1-4-7-10 or 1-9-17, etc.; AP bursts were tested in any given cell pair, for a total of $n=99$ combinations). As shown in Figure $1 C(n=28$ pairs $)$, even as low as $2-5$ APs resulted in measurable postburst potentiation. Single exponential fits to the measured potentiation predicted that the amplification would reach $50 \%$ of its maximal value with a theoretical $2.92 \mathrm{AP}$ burst ( $\pm 5 \%$ confidence range: $2.29-3.7 \mathrm{APs}$; fit $R^{2}=0.966, p=$ $\left.3.8 \times 10^{-10}\right)$. A single burst with 5 APs activated $68.9 \pm 8 \%$ of the maximal potentiation, and $10 \mathrm{AP}$ bursts increased this value to $90.1 \pm 10.2 \%$. Control pulses alone (i.e., omitting the bursts from the protocol) did not evoke potentiation (1.05 \pm 0.12 ; Fig. $1 C, 0$ AP burst point). These data demonstrate that physiological GC AP bursts are well within the dynamic range for the induction of this form of MF plasticity in FF-INs. Interestingly, the effects of shorter bursts also lasted for several seconds (Fig. 1C, inset).

Next, to explore the frequency-dependency of the single burst-induced potentiation, we evoked 8 presynaptic APs at 20, 40, 80, and $160 \mathrm{~Hz}$ in the same FF-IN-pairs ( $n=8$ pairs; Fig. $1 D)$. These presynaptic stimulation protocols did not include preburst control pulses, and the postburst amplitudes were compared with the first responses during the bursts. Interestingly, the amplification showed only a moderate frequency dependence (linear fit, $R^{2}=0.769, p=0.08$ ).

Finally, we stimulated presynaptic GCs using bursts that had been observed in in vivo recordings previously by other laboratories (Henze et al., 2002; Pernía-Andrade and Jonas, 2014; Diamantaki et al., 2016). These bursts included 4-6 APs with average firing from 100 to $175 \mathrm{~Hz}$. The timing of the spikes within the burst either precisely replicated the in vivo data (pattern 1) (Henze et al., 2002) or, if the exact timing of each AP within the actually observed bursts was not available, the bursts of spikes were patterned to reflect the previously reported average frequencies and jitter by considering the approximate frequencyadaptation during bursts: pattern 2 (Pernía-Andrade and Jonas, 2014) and pattern 3 (Diamantaki et al., 2016) (Fig. 1E, inset).
These in vivo bursts and the burst-protocol that we used above (6 APs at $150 \mathrm{~Hz}$ ) evoked similar potentiation in the same FF-INpairs (Fig. $1 E ; n=8$ pairs, $3 \mathrm{~s}$ after burst, $p=0.82, F_{(3,21)}=0.306$, one-way repeated measure ANOVA, sphericity assumed).

For the subsequent experiments described below, unless specifically stated otherwise, the more robust 15 AP presynaptic bursts were used to enable us to investigate various aspects and mechanisms of postburst potentiation in FF-INs.

\section{Postsynaptic cell type specificity of the postburst MF-EPSC potentiation}

Next, we tested whether MF inputs to other postsynaptic cell types in the CA3 network, including pyramidal cells and nonFF-IN GABA cells, also exhibit strong potentiation after a single short MF burst. In CA3 pyramidal cells, the postburst test responses were increased compared with the preburst controls (1.72 \pm 0.26 -fold increase, $n=12$ pairs, total number of postburst test responses in all pairs at various postburst times: $n=25$; $p=0.01, t_{(24)}=2.798$; Fig. $\left.2 A, B\right)$, but the potentiation was less robust than the tripling of MF-EPSCS observed in FF-INs (compare Fig. $2 B$ with Fig. $1 B$; the data in Fig. $2 B$ were obtained using either 6 or 15 AP bursts because, in 4 of the tested pyramidal cell pairs, the 15 AP presynaptic MF bursts resulted in spiking of the postsynaptic pyramidal cell even in voltage clamp; control experiments showed that the postburst potentiation in pyramidal cells remained smaller than in interneurons even after 20 AP bursts; Fig. 2A). The difference in the nature of the postburst potentiation between the two cell groups was especially striking when the increase in the test response was expressed with respect to the magnitude of the short-term facilitation of the MF-EPSCs taking place during the bursts. As shown in Figure $2 C$, $D$, when normalized to the maximum responses during the burst, the postburst test responses were significantly larger in the FF-INs compared with the pyramidal cells. These results suggest that the postsynaptic responses in CA3 pyramidal cells are more sensitive to the bursts, while they are relatively more affected during the postburst period in FF-INs.

Because the postburst MF-EPSC potentiation was not different between axo-axonic, PV and CCK basket, and ivy cells, we next examined whether the similarity of the effects of single MF bursts extended to non-FF-IN interneurons. The SLCs have negligible local, within-CA3 axons and thus do not participate in feedback or feedforward inhibition but comprise a part of the hippocampo-septal GABAergic projection (Gulyás et al., 1992; Spruston et al., 1997; Jinno et al., 2007). Interestingly, despite the fact that SLCs receive excitatory inputs almost exclusively from MFs (Wittner et al., 2006), the MF synapses on SLCs have extremely low initial release probability, which increases only after sustained presynaptic activity (Szabadics and Soltesz, 2009; Szabadics et al., 2010). Our paired recordings showed that MF synaptic release onto SLCs was increased shortly after the 15 AP MF bursts (Fig. $3 A, D$ ). However, in contrast to FF-INs, the amplitudes of the postburst test MF-EPSCs remained small compared with the compound EPSC amplitudes evoked during the bursts and quickly returned to control baseline (Fig. 3). Thus, the bursts were not able to induce the sustained, seconds-long potentiation of MF-EPSCs in SLCs. Importantly, similarly small burst effects were observed, even when the unusually low control synaptic release probability of the MF-SLC connections (Fig. 3B) was artificially increased (Fig. $3 C, D$ ), suggesting that the postsynaptic cell type specificity of the amplification was not simply a consequence of the different initial release probabilities of the MF inputs onto the SLCs and FF-INs. 
A

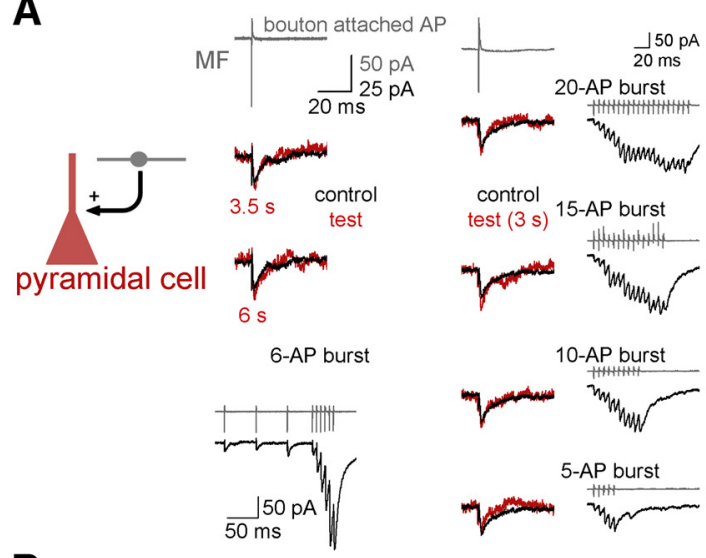

B

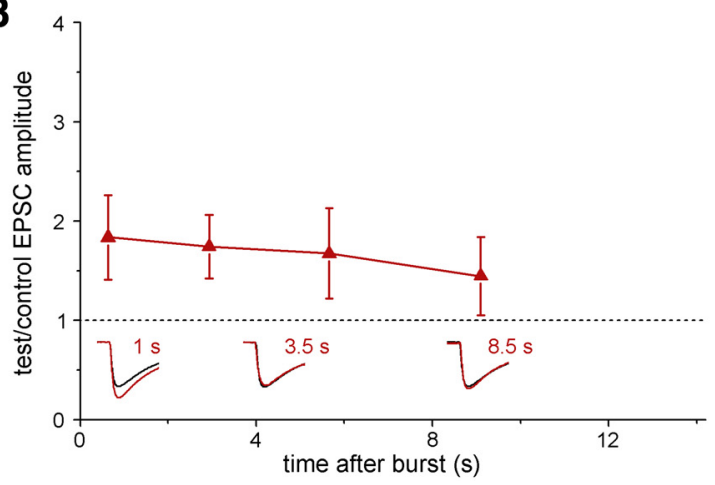

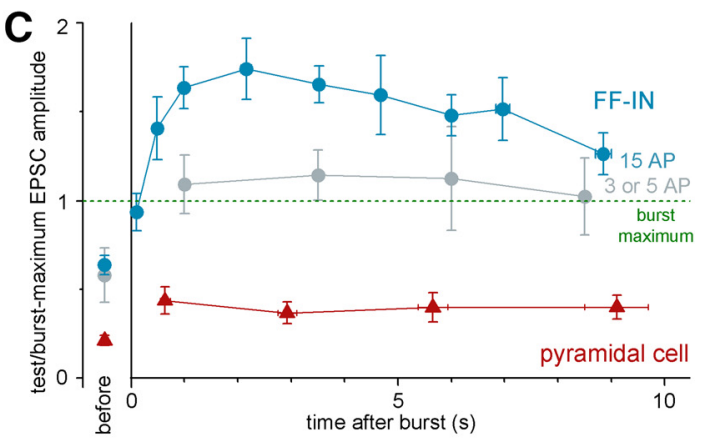

D
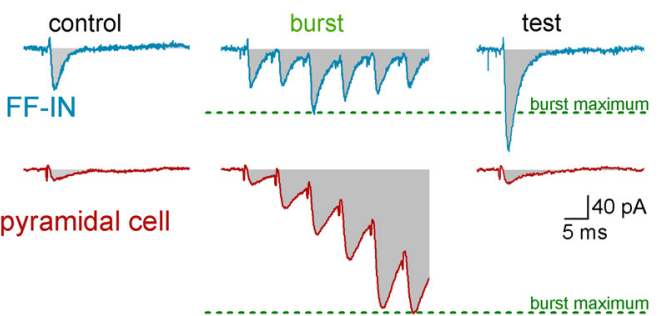

pyramidal cell
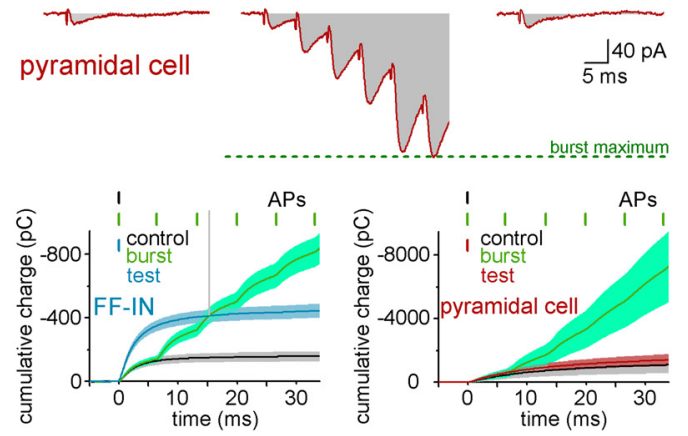

Figure 2. Postsynaptic cell type specificity of postburst potentiation of MF-EPSCS. A, Single presynaptic MF bursts, consisting of various numbers of APs, resulted in small postburst potentiation effects on the EPSCs of a representative MF-pyramidal cell pair. Traces represent presynaptic MF terminal APs in bouton-attached recordings (gray) and the postsynaptic responses before and after single burst. Bursts and postsynaptic responses (including preburst control and postburst test EPSCS, and during-burst responses) are shown next to the corresponding traces. $\boldsymbol{B}$, Time course of the effects of single, brief presynaptic bursts ( 6 or $15 \mathrm{AP}, 150 \mathrm{~Hz}$ ) on subsequent single-AP-evoked MF-EPSCs in postsynaptic CA3 pyramidal cells ( $n=46$ data points from $n=12$ pairs; compare with Fig. 1B). Inset, Example control and test MF responses at different postburst delays. Anatomy of the postsynaptic pyramidal cell and presynaptic DG GC (back-labeled via the MF terminal recording) is shown in Figure 2-1 (available at https://doi.org/10.1523/JNEUROSCI.1595-17.2018). C, Summary graph showing the amplitude of the postburst test responses in pyramidal cells and FF-INs relative to the compound maximal amplitudes reached during the bursts (dashed line) in the same synaptic inputs (red represents pyramidal cells, $n=46$ data points from $n=12$ pairs; blue represents FF-INs with $15 \mathrm{AP}$ bursts; light blue represents FF-INs with short, 3 or $5 \mathrm{AP}$ bursts, $n=10$ pairs; the same responses were reanalyzed as in Fig. $1 B, C$. The relative amplitudes of the preburst control responses ("before") are shown separately. D, Comparison of MF-EPSCs before, during, and after single presynaptic MF bursts in a representative FF-IN (blue traces) and pyramidal cell (red). Green dashed lines indicate maximum amplitude of the compound EPSCs during the burst for comparison. Gray areas represent the integral areas (charge transfer). Bottom, Graphs represent the activity- and postsynaptic cell type-dependent differences of the time course of charge transfer.

\section{Presynaptic origin of the burst-induced sustained amplification in FF-INs}

To better understand the underlying synaptic mechanisms of the single-burst-induced amplification, we tested whether the amplification of the MF-responses in FF-INs was initiated by presynaptic or postsynaptic changes. The probability of failures decreased after single bursts (from $53.3 \pm 3 \%$ to $25.0 \pm 3 \%$; $p=$ $3.5 \times 10^{-20}, t_{(67)}=13.12, n=68$ FF-IN pairs, paired $t$ test; Fig. $4 A$ ), consistent with presynaptic changes. The rise times and halfwidths of the postburst test MF-EPSCs remained unchanged compared with preburst control $(625 \pm 38 \mu$ s vs $583 \pm 31 \mu \mathrm{s}, p=$ $0.25, t_{(50)}=1.155 ; 2.46 \pm 0.12 \mathrm{~ms}$ vs $2.61 \pm 0.09 \mathrm{~ms}, p=0.13$, $t_{(51)}=-1.556$, paired $t$ test; Fig. $\left.4 B\right)$. Comparison of the shortterm plasticity of the control and test responses evoked using 3 MF APs at $20 \mathrm{~Hz}$ before and after the burst was also consistent with a presynaptic locus for the postburst MF-EPSC potentiation. Specifically, the PPR decreased after the burst and remained small (i.e., more pronounced depression) for several seconds (Fig. 4C), similar to the time course of the burst-induced MFEPSC potentiation in Figure $1 B$.

The PPR of the control (initial, preburst) MF responses varied over a relatively wide range across FF-IN pairs (even within the same types) under baseline conditions. The control PPR showed a correlation with the magnitude of the potentiation $\left(R^{2}=0.438\right.$, slope: $\left.1.54 \pm 0.12, F_{(1,196)}=154.8, p<10^{-20}\right)$, indicating that, for connections whose release probability was initially low (i.e., high PPR), single bursts led to larger potentiation, whereas the relative potentiation of the more reliable connections was rather small (Fig. 4D). The postburst test PPRs did not correlate with the initial control PPRs of the same connections $\left(R^{2}=0.035\right.$, slope: $\left.0.139 \pm 0.048, F_{(1,196)}=8.227, p=0.0046\right)$, primarily because the PPRs after the bursts were invariably low (i.e., highly reliable, depressing responses), suggesting that single bursts result in a high-release state in MF-FF-IN synapses regardless of their initial reliability.

\section{Investigations into plasticity mechanisms}

To characterize postburst plasticity in FF-INs further, we first explored whether the postburst potentiation at unitary MFFF-IN synapses was present at a lower extracellular $\mathrm{Ca}^{2+}$ concentration (1.3 mM) that may be more physiological (Rancz et al., 2007; Lorteije et al., 2009) than the one used in the standard recording solution $(2 \mathrm{~mm})$ in the above-described experiments. In $1.3 \mathrm{~mm}$ extracellular $\mathrm{Ca}^{2+}\left(\mathrm{Mg}^{2+}\right.$ concentration was kept unchanged at $2 \mathrm{~mm}$ ), as expected, the control response amplitude decreased and PPR increased $\left(n=8\right.$ pairs, $p=0.012, t_{(7)}=-3.38$ 

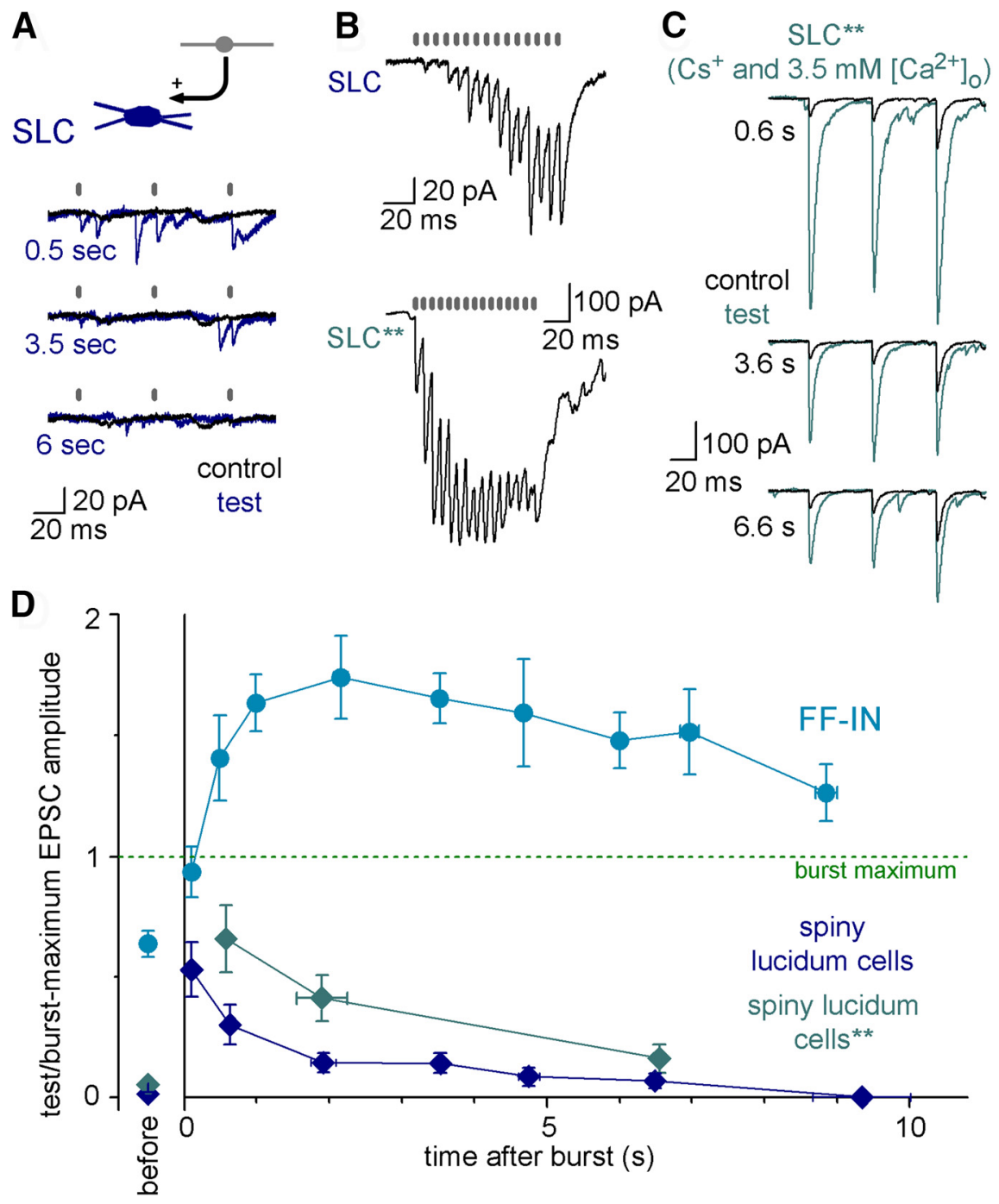

Figure 3. Effects of single MF bursts on the unitary MF responses evoked in SLCs, which are GABAergic but are not considered to be FF-INs. $A$, Control and postburst test MF-EPSCs from an example CA3 GC-SLC pair at three different time points after single presynaptic bursts. Gray bars represent APs, often weak responses among the frequent spontaneous events. $\boldsymbol{B}$, The MF responses of two SLCs during the burst (15 APS at $150 \mathrm{~Hz}$ ) in standard solutions (top) and in the presence of presynaptic intracellular CSCl (by replacing $40 \mathrm{~mm} \mathrm{KCl}$ ) and $3.5 \mathrm{~mm}$ extracellular $\mathrm{Ca}^{2+}$ level (bottom, $\left.{ }^{* *}\right)$. C, A representative experiment, in which reliable synaptic transmission was obtained in a CA3 GC-to-SLC connection by the above modifications in the recording conditions. $\boldsymbol{D}$, Summary graph showing the amplitude of the postburst responses in SLCs relative to the compound maximal amplitudes during the bursts. Dark blue represents SLC with standard presynaptic recording ( $n=61$ data points from $n=20$ pairs). Gray represents SLC with artificially elevated MF release ( $n=15$ data points from $n=5$ pairs). Blue curve indicates data for $\mathrm{FF}$-INs replotted from Figure $2 C$ to highlight the robust differences between the two GABAergic cell groups. The relative amplitudes of the control responses before the bursts are shown separately. For the anatomy of the presynaptic and postsynaptic cells, see Figure 3-1 (available at https://doi.org/10.1523/JNEUROSCl.1595-17.2018).

and $0.011, t_{(7)}=-1.97$, respectively, paired $t$ test), but the postburst potentiation of the MF-FF-IN inputs not only persisted but increased (from $1.81 \pm 0.16$ test/control amplitudes to $3.55 \pm$ 0.48 in the same pairs with normal and low $\mathrm{Ca}^{2+}$ conditions, respectively, $p=0.002, t_{(12)}=-3.862$, paired $t$ test; Fig. $\left.5 A\right)$. The apparently larger postburst potentiation in lower extracellular $\mathrm{Ca}^{2+}$ was due to a larger decrease of the control EPSCs compared with the postburst EPSCs $(-76.4 \pm 1.9 \%$ vs $-54.3 \pm 5.8 \%, p=$ $0.0017, t_{(12)}=-4.034$, paired $t$ test), consistent with a presynaptic origin for the plasticity. Next, we tested the effects of presynaptic $\mathrm{Ca}^{2+}$ chelation on postburst plasticity. Presynaptic loading of the $\mathrm{Ca}^{2+}$ chelator EGTA (0.5-2.5 mM; the final concentration in the presynaptic terminal was unknown, as the CA3 GC somatic recording site to presynaptic terminal distance varied) (Szabadics et al., 2010) strongly reduced the control response $\left(p=4 \times 10^{-4}, t_{(15.03)}=4.615\right.$, paired $t$ test) but did not prevent the single-burstinduced amplification $\left(p=0.0039, t_{(15)}=\right.$ 3.409, $t$ test; Fig. $5 B$ ). Therefore, postburst potentiation at MF-FF-IN synapses persisted at physiological extracellular $\mathrm{Ca}^{2+}$ levels and was consistent with the data shown in Figure 4D. In addition, consistent with the results of the $1.3 \mathrm{mM} \mathrm{Ca}{ }^{2+}$ and EGTA experiments, when the extracellular $\mathrm{Ca}^{2+}$ concentration was elevated to $5 \mathrm{~mm}$, the baseline responses increased by $178 \pm$ $33 \%$, whereas the effect on postburst EPSPs was smaller at $104 \pm 28 \%\left(p=0.012, t_{(14)}=\right.$ 2.888 , paired $t$ test, data not shown).

Next, we investigated whether the delay between the presynaptic spike and the onset of the postsynaptic response in FFINs was altered by the single bursts of MF APs. Our analysis showed that the delay of the test responses was significantly shorter than the delay of the control responses in MF-FF-IN pairs (control: $0.949 \pm 0.023$ ms; test: $0.868 \pm 0.023 \mathrm{~ms}, n=57$ responses, $p=9 \times 10^{-12}, t_{(56)}=8.565$, paired $t$ test; Fig. $5 C$, top). Furthermore, the variance of the delays was also significantly decreased (from $0.0294 \pm 0.0023$ $\mathrm{ms}^{2}$ to $0.018 \pm 0.0018 \mathrm{~ms}^{2}, p=3 \times 10^{-5}$, $t_{(56)}=4.544$; Fig. $5 C$, bottom; the CV of the delay also changed: from $0.176 \pm$ 0.008 to $0.145 \pm 0.008, p=4.5 \times 10^{-4}$, $\left.t_{(56)}=3.729\right)$. Therefore, the single, brief burst of MF APs results in accelerated, more precise, and more reliable release.

In subsequent experiments, we tested whether the burst-induced plasticity could be occluded by the phorbol ester $\mathrm{PDBu}$, thought to promote vesicle priming and saturate the capacity of the release machinery (Rhee et al., 2002; Lou et al., 2008; Fioravante et al., 2014; Taschenberger et al., 2016). PDBu (1 $\mu \mathrm{M})$ increased the control response amplitude before the bursts (from $-39.6 \pm 5.3 \mathrm{pA}$ to $-146.3 \pm 52.2 \mathrm{pA}, p=0.011, t_{(12)}=$ 2.646) and decreased the PPR (from $1.51 \pm 0.10$ to $0.61 \pm 0.04, p=0.0004, t_{(12)}=4.462, t$ test $)$, and there was no significant burst-induced potentiation of the already enhanced MF-EPSCs in FF-INs in the presence of PDBu (test/ control amplitude: $1.32 \pm 0.15, p=0.21, t_{(12)}=2.11, t$ test; Fig. $5 D)$. Furthermore, single MF bursts failed to potentiate the test responses with respect to the preburst control responses in the presence of $\mathrm{PDBu}$, even in reduced extracellular $\mathrm{Ca}^{2+}(1-1.6$ $\mathrm{mM})$, applied to compensate for the amplitude-enhancing effects of PDBu (test/control amplitude: $1.4 \pm 0.15, p=0.19, t_{(15)}=$ 2.67; the reduced $\mathrm{Ca}^{2+}$ concentration alone would have been expected to increase amplification, see Fig. $5 A$ ). In addition, application of $\mathrm{PDBu}$ also prevented the bursts from accelerating the synaptic release (onset delay of the control and test responses: 
before PDBu: $1.005 \pm 0.069 \mathrm{~ms}$ and $0.944 \pm 0.082 \mathrm{~ms}, p=0.017, t_{(8)}=3.01$, paired $t$ test; in PDBu: $0.96 \pm 0.07 \mathrm{~ms}$ and $0.967 \pm 0.084 \mathrm{~ms}, p=0.87, t_{(8)}=0.165$; ANOVA one-way repeated measure, multivariate test: $p=0.048, F_{(6)}=4.851$, between-subject effects: $p=9.7 \times 10^{-7}$, $F_{(1,8)}=177.01$; Fig. 5D, bottom right). Together, these results suggested occlusion, and thus at least a certain degree of mechanistic convergence, between the burst and $\mathrm{PDBu}$ effects, perhaps in the form of enhanced vesicle priming at MF synapses on FF-INs.

We also investigated whether mechanisms implicated in other forms of plasticity at MF synapses were involved in the postburst potentiation described in this paper. PKC and Munc13 proteins are known to be involved in regulating release at many synapses (Betz et al., 1998; Augustin et al., 1999; $\mathrm{Wu}$ and $\mathrm{Wu}, 2001$; Rosenmund et al., 2002; Junge et al., 2004; Korogod et al., 2007; Wierda et al., 2007; Fioravante et al., 2011; Chu et al., 2014), including MFs (Alle et al., 2001; Pelkey et al., 2005; Hainmüller et al., 2014). In agreement with these results, various direct and indirect inhibitors of PKC activity (calphostin-C, intracellular PKC1936, GF109203X, and U73122, the latter inhibits PLCs that generate diacyl-glycerol for PKC functions) were able to decrease the control EPSCs at MF-FF-IN synapses. Specifically, acting as positive controls, the drug application reduced the control EPSC amplitudes (calphostin-C, with respect to predrug: $60.5 \pm 27.4 \%$; U73122: to $37.5 \pm 17.5 \%)$ and increased the PPR (by $0.53 \pm 0.55$ and $0.67 \pm 0.37$, respectively; Fig. $6 A$ ); similarly, in the case of experiments with PKC19-36 fragment and GF109203X (these drugs were preincubated), the EPSC amplitudes were $37.9 \pm 3.2 \%$ and $48.6 \pm 5 \%$, respectively, of the untreated control pairs, and the PPRs were increased by $0.32 \pm 0.26$ and $0.32 \pm 0.11$, respectively. In contrast, another type of PKC blocker, Go6976, did not alter the control MF responses (control EPSC amplitude with respect to predrug control: $108.6 \pm 55.2 \%$; PPR: $0.24 \pm$ $0.47)$.

Importantly, if PKC activity were necessary for the burst-induced potentiation, these various PKC inhibitors should have decreased or eliminated the postburst plasticity. However, the postburst potentiation was not affected in the presence of any of these inhibitors (Fig. 6B, group of bars under the label "PKCs"), despite the presence of positive control effects for most of the drugs. Because Munc13 pro-
A
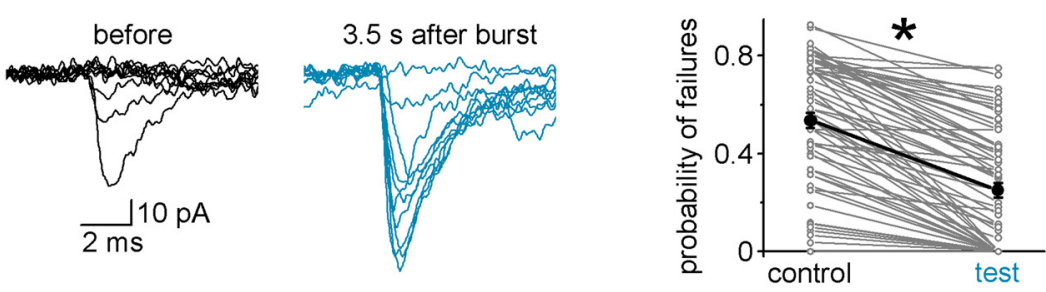

B
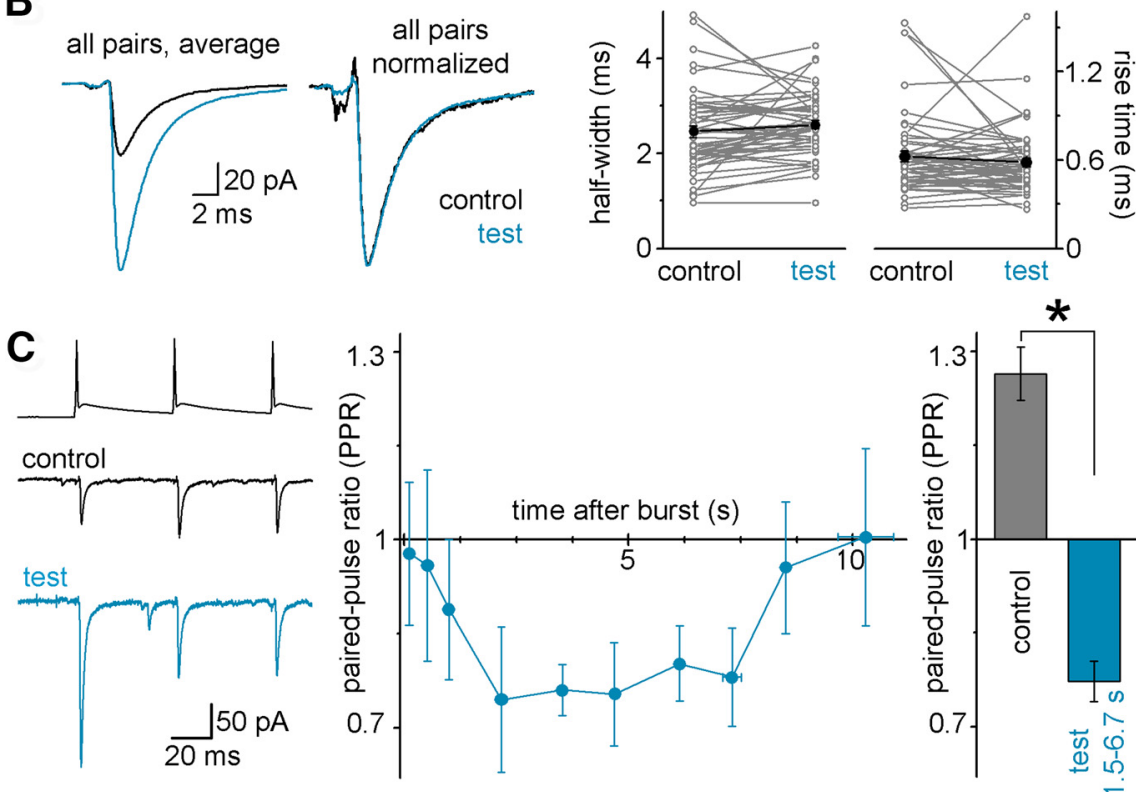

D

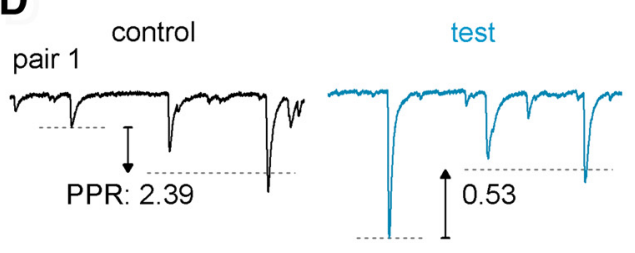

pair 2
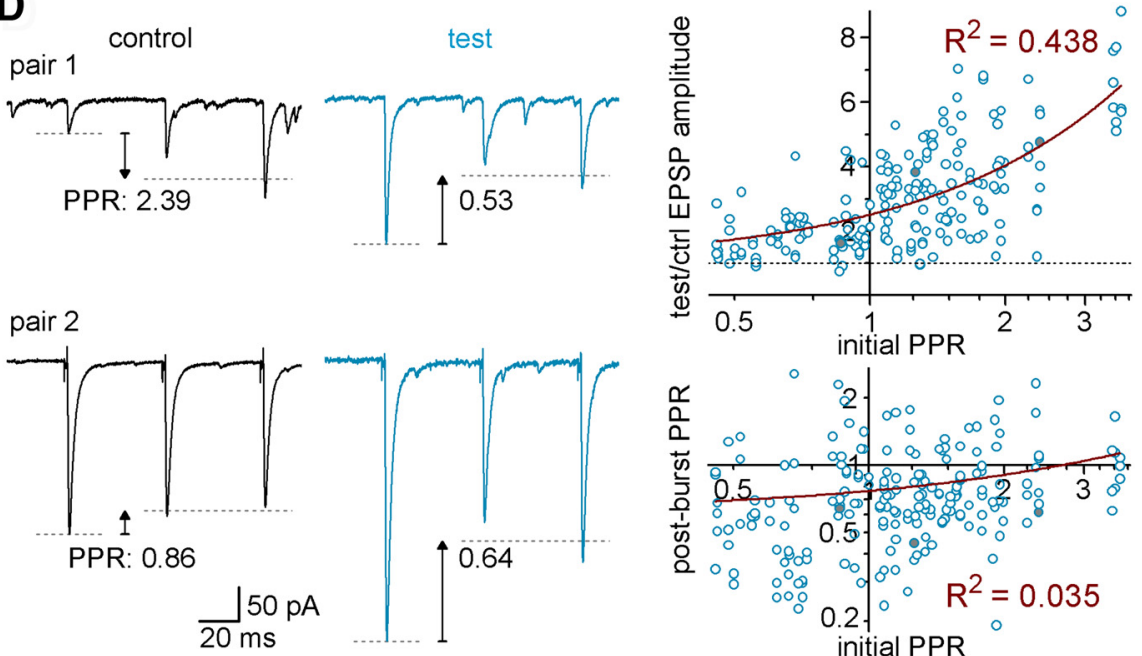

Figure 4. Presynaptic origin of the burst-induced potentiation in FF-INs. $\boldsymbol{A}$, The probability of synaptic response failures decreases after single presynaptic MF bursts. Representative traces of a CA3 GC-CCK ${ }^{+}$basket cell connection before (black) and after (blue) single bursts (10 traces from each condition). Right, Connected gray symbols represent the failure rates in individual pairs with identified FF-INs ( $n=68$ pairs) during control and test MF stimuli (1.5-6.7 s postburst delays). Black represents the average data from all FF-IN connections. The failure rates were analyzed from at least 10 trials for each condition. $\boldsymbol{B}$, Actual and normalized average EPSCs evoked in FF-INs ( $n=51$ pairs) by single MF APs before and 1.5-6.7 s after single presynaptic bursts. The events were aligned to the presynaptic AP peak. Graphs represent the half-width and 10\%-90\% rise times of the MF responses in individual FF-INs (gray) and their average (black), which were similar before and after the bursts. C, Example traces demonstrating changes in short-term plasticity of the MF-EPSCs in an IvyC evoked by 3 APs before and $6 \mathrm{~s}$ after bursts (control PPR: 1.26; test PPR: 0.45). Summary plot shows data from all in identified postsynaptic FF-INs; the PPR (calculated as the ratio of the average of the second and third amplitudes to the first amplitude) was low during the time when the EPSCs were potentiated. Summary bar graphs represent PPRs of all FF-IN connections before (control) and 1.5-6.7 s after the burst (test). D, Example traces: from IvyC MF pairs; note the similarity of test PPRs (at 6 s postburst) despite the different control PPRs. Right panels: Top, Correlation between the control (initial) PPR and the magnitude of potentiation (linear fit, maroon line; note the logarithmic scaling of the PPR axes). Bottom, Independence of postburst test PPR of the control PPR. Each circle represents individual test responses from identified FF-IN pairs. Circles with gray fillings indicate the representative pairs illustrated in $\boldsymbol{C}$ and $\boldsymbol{D}$. ${ }^{*}$ marks significant difference between control and test responses (see text for details). 
A
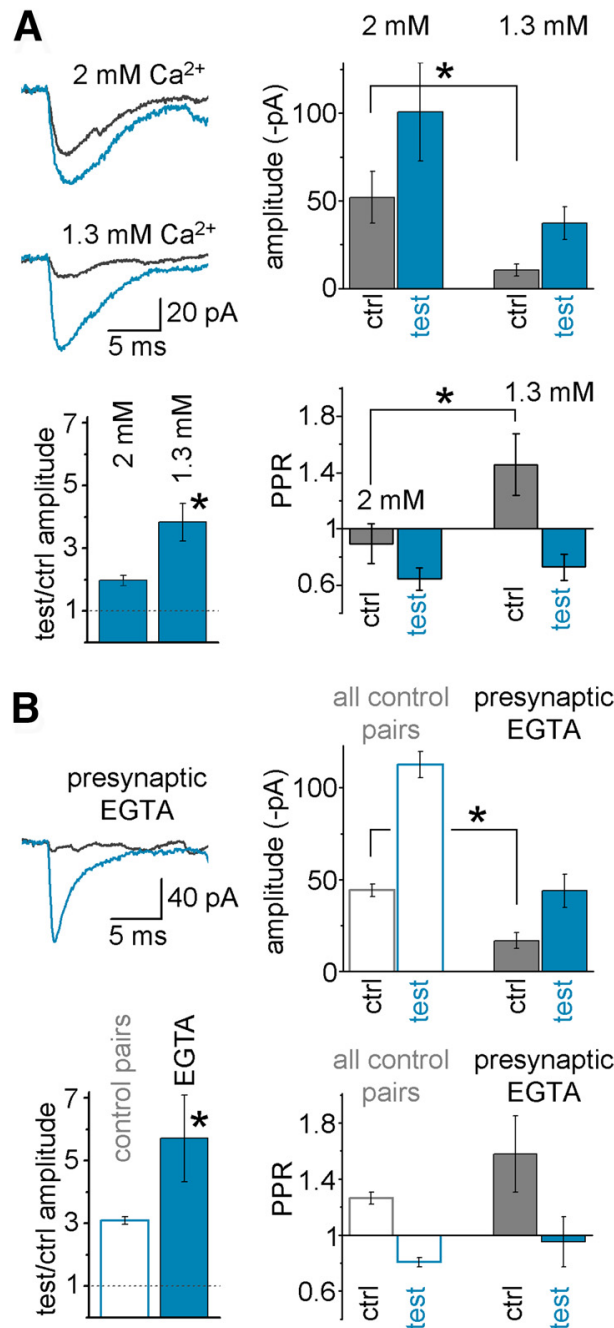
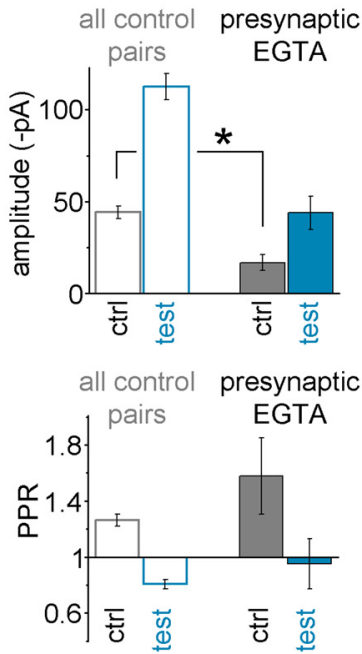

C
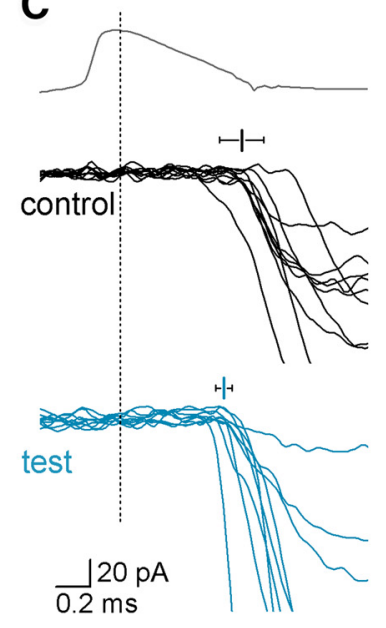

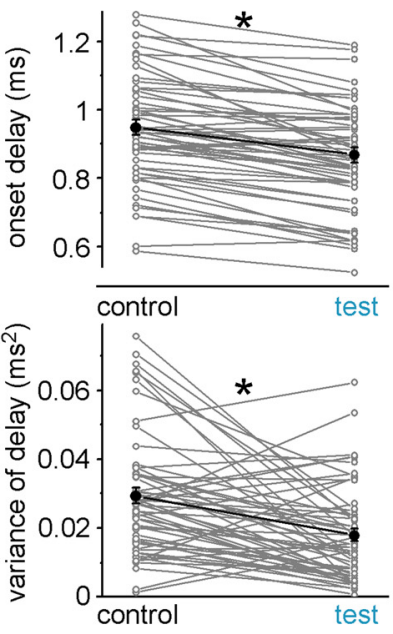

D
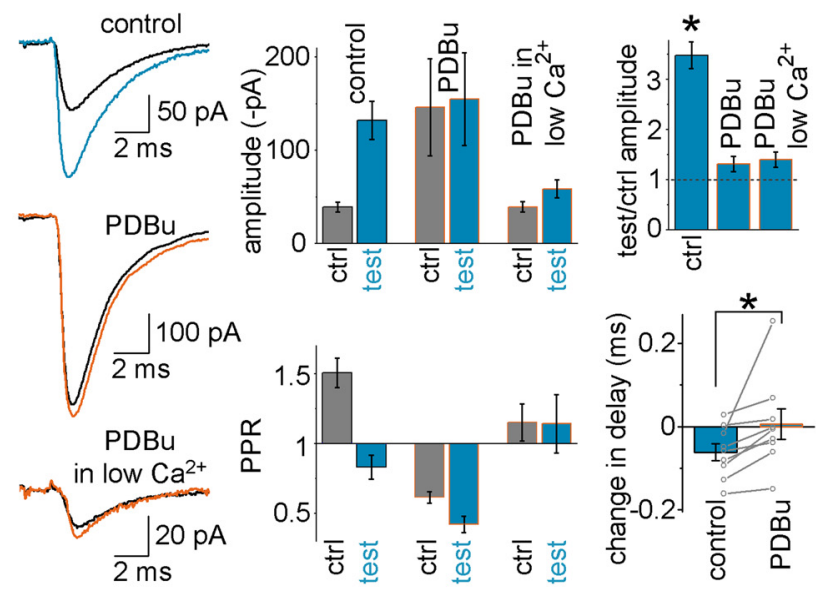

Figure 5. Investigations into mechanisms of postburst plasticity in FF-INs. A, Persistence of the postburst potentiation in lower (1.3 mm; allegedly physiologically more relevant) extracellular $\mathrm{Ca}^{2+}$ levels. Example traces are shown from a CA3 GC-IvyC pair in which the effects of single presynaptic bursts were first tested in the presence of standard $2 \mathrm{~mm} \mathrm{Ca}{ }^{2+}$ concentrations and then in $1.3 \mathrm{~mm} \mathrm{Ca}{ }^{2+}$ (preburst control responses: black; test responses: blue, $3.6 \mathrm{~s}$ after burst). Bar graphs represent average data from all pairs $(n=8)$; the lower $\mathrm{Ca}^{2+}$ concentration decreased the response amplitudes and increased the PPRs, but the potentiation persisted. $B$, Example traces from a CA3 GC-AAC pair in which the effects of single presynaptic bursts were tested while the presynaptic CA3 GC was recorded with $1 \mathrm{~mm}$ intracellular EGTA to chelate $\mathrm{Ca}^{2+}$. Bar graphs represent average data from all presynaptic EGTA pairs $(n=8)$. Presynaptic EGTA strongly decreased the control responses, but the burst-induced potentiation persisted. C, Example traces illustrate the accelerated and more precise release before and after single presynaptic bursts from the same pair; average presynaptic APs and individual EPSCs are shown; mean and variance of the delay are also indicated, with error bars; failures were excluded for clarity. Right panels, Plots of the mean and variance values of the synaptic onset delay in each MF-FF-IN pair before and after single bursts (connected gray symbols) and their average (black). $D$, The DAG analog phorbol ester PDBu ( $1 \mu \mathrm{m}$ ), which promotes vesicle priming, increased MF-EPSC amplitude before bursts (note the different scale bars) and prevented burst-induced potentiation in a representative pair. Subsequent reduction of the release probability following the application of decreased extracellular $\mathrm{Ca}^{2+}(1 \mathrm{~mm})$ in the PDBu-containing perfusing solution significantly reduced the average amplitudes, but burstinduced amplification remained negligible in the same pair. Summary bar graphs show that bursts were no longer effective in eliciting potentiation in the same pairs in the presence of PDBu regardless of the release probability; furthermore, PDBu also prevented the burst-induced decrease in the delay of the responses (bottom right). Symbols represent changes in synaptic delays in control conditions and in the presence of PDBu. Bars represent average data. ${ }^{*}$ marks significant difference.

teins share both active domains (which are targeted by calphostin-C, $\mathrm{PDBu}$, and native diacyl-glycerol sources) and synaptic functions with PKCs (promoting release), we cannot exclude the possibility that Munc13s were involved in the positive control effects, but it is also unlikely that they were necessary for the burst-induced potentiation. Similarly, we were unable to block the postburst potentiation in FF-INs using the PKA inhibitors KT5720 and presynaptically loaded PKI6-22 fragment (Fig. 6B, bars under the label "PKA"; the lack of an effect on the postburst potentiation was in the presence of positive control effects on the control EPSC amplitudes: KT5720, $51.2 \pm 17.8 \%$; and PPR, $0.24 \pm 0.25)$. In addition, neither activation nor inhibition of mGluR7 and mGluR2/3 occluded or eliminated the postburst potentiation (Fig. 6B, bars labeled
"mGluRs") (AMN082, control effect on EPSC and PPR, respectively: $70.2 \pm 33.7 \%, 0.51 \pm 039$; MSOP, $62 \pm 21.7 \%$, $-0.18 \pm 0.17$; BINA, $78.6 \pm 9.8 \%,-0.09 \pm 0.12$; and DCGIV, $11.3 \pm 4 \%, 0.41 \pm 0.38$; mGluR7 is specifically expressed at MF synapses on GABAergic cells) (Shigemoto et al., 1997; Pelkey et al., 2008). The fact that none of these pharmacological manipulations of PKC, PKA, and mGluRs was able to significantly modify postburst potentiation (note the remarkably stable level of potentiation in the presence of various drugs in Fig. $6 B$ ) suggested that the single, brief burst-induced MF plasticity at FF-IN synapses described in this study may involve a potentially noncanonical molecular pathway that will need to be identified in the future. 

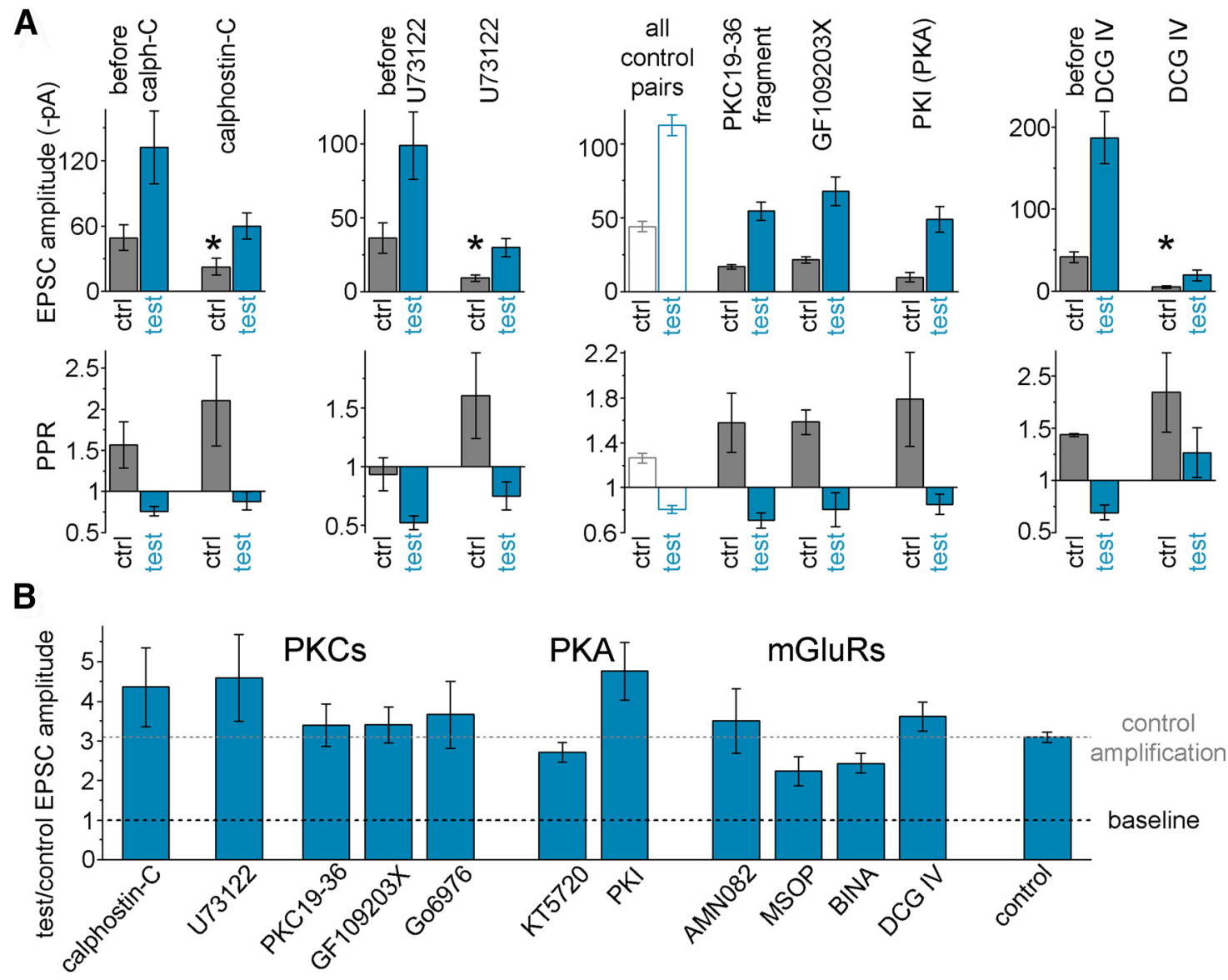

Figure 6. Summary of the pharmacological experiments aimed at probing the potential involvement of various plasticity pathways in the postburst potentiation in $\mathrm{FF}-\mathrm{INs}$. $A$, Calphostin- $\mathrm{C}(1 \mu \mathrm{M}$, $n=12)$ is a PKC (and Munc13) inhibitor that acts on DAG-binding domains. U73122 $(2.5 \mu \mathrm{M}, n=8)$ is a phospholipase C inhibitor. Both inhibitors affected baseline release as indicated by the attenuation of the control response amplitudes indicating the involvement of these pathways in the regulation of release at these synapses, serving as positive internal controls indicating drug effectiveness. PKC19-36 (100 $\mu \mathrm{m}, n=7$, synthetic autoinhibitory domain, applied intracellularly) and GF109203X ( $1 \mu \mathrm{m}, n=11$, acts on the ATP binding site; slices were preincubated for at least $60 \mathrm{~min}$ ) are selective PKC inhibitors. The small control amplitudes and large PPRs provided positive internal controls for these inhibitors. The averages of all control responses are also shown for comparison (because these inhibitors required intracellular application and pretreatment). DCG IV is an mGluR2/3 agonist $(1 \mu \mathrm{M}, n=7)$ that selectively inhibits MF responses. ${ }^{*}$ marks significant difference in the control (before burst) EPSC amplitudes in control conditions and after drug application. B, Average amplification at 1.5- $6.7 \mathrm{~s}$ after single $15 \mathrm{AP}$ bursts in the presence of various pharmacological agents. In addition to the above drugs, the burst-induced amplification of MF-EPSCs is shown in the presence of G06976 ( $0.25 \mu \mathrm{M}, n=8$, a subtype-selective PKC inhibitor), KT5720 ( $200 \mathrm{~nm}, n=10$, a PKA inhibitor that works by competing with ATP binding; PKAs are involved in pathways that regulate presynaptic release, including RIM proteins, whose PKA-dependent phosphorylation promotes vesicle priming and PKAs are also involved in posttetanic potentiation mechanisms at MF synapses onto DG inhibitory cells) (Alle et al., 2001), PKI (2.5 $\mu$ m intracellularly, PKA inhibitory fragment $6-22$ amide, that binds to the substrate site) (Hashimotodani et al., 2017), AMN082 ( $1 \mu \mathrm{m}, n=6$, selective mGluR7 agonist), MSOP (150 $\mu \mathrm{m}, n=8$, selective inhibitor of Group III mGluRs including mGluR7), or BINA (5 $\mu \mathrm{m}, n=12$, selective positive allosteric modulator of mGluR2). For additional positive control effects, see Results. Postburst potentiation in the absence of these drugs ("control amplification") is also shown for comparison. None of these pharmacological modifiers of known plasticity pathway components was able to significantly block the postburst potentiation.

\section{Seconds-long potentiation of FF-IN activity in the CA3 network after individual MF bursts}

Our results suggest that FF-INs and pyramidal cells in the CA3 area respond to single MF bursts differently, with weak (strong) short-term facilitation of the unitary EPSCs but strong (weak) postburst potentiation in FF-INs (pyramidal cells) (Fig. 2B-D). In other words, the seconds-long MF-EPSC plasticity in FF-INs appear to reflect the recent passage of a MF burst, whereas changes in MF-EPSCs in pyramidal cells preferentially take place during the MF bursts themselves. To test these ideas further, in the final series of experiments, we examined whether a single brief burst of APs in an individual MF is able to increase FF-IN activity in the network by measuring the incidence of diIPSCs in pyramidal cells that were not themselves synaptically connected to the activated MF (for synaptic delays and kinetics of diIPSCs after APs in the MF, see Fig. 7E) (Neubrandt et al., 2017). The probability of diIPSCs was modestly but significantly increased in randomly selected pyramidal cells during the bursts of APs in individual MFs (Fig. $7 A$, $B$, bar labeled "burst"; diIPSCs after APs in a single MF: control responses: $11.9 \pm 3.4 \%$, during bursts: $18.1 \pm 1.9 \%, p=0.0029, t_{(23)}=-3.329$, paired $t$ test, $n=24$ pairs of MF and randomly chosen pyramidal cell). In contrast, in the same pairs, the probability of diIPSCs almost tripled for several seconds following the AP burst in the individual MF, with a time course that showed remarkable similarity to the potentiation of unitary MF-EPSCs after bursts (compare Fig. $7 B$ with Fig. $1 B$; probability of diIPSCs in response to test pulses $1.5-6.7 \mathrm{~s}$ after the bursts: $29.4 \pm 3.1 \%, p=1 \times 10^{-9}, t_{(39)}=-7.944$, compared with control preburst responses and $p=2 \times 10^{-4}, t_{(39)}=-4.09$, compared with diIPSCs during the bursts, paired $t$ test). These data indicated that a single burst in an individual MF enhanced the probability of IPSCs for several seconds in a population of pyramidal cells that were not directly connected to the activated MFs, presumably through the involvement of one or more unre- 


\section{A}

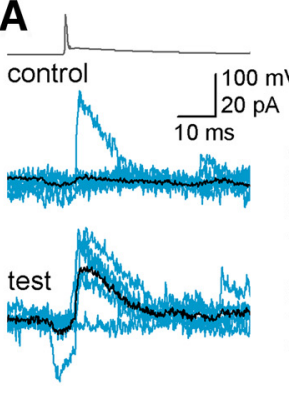

C

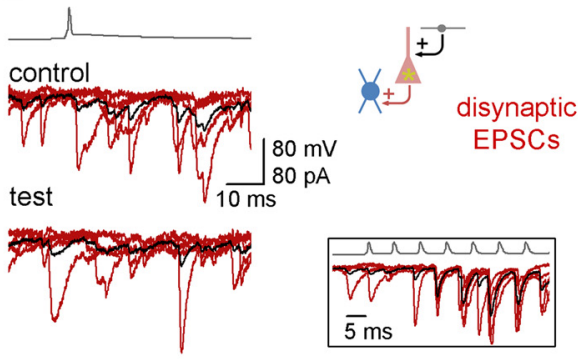

B

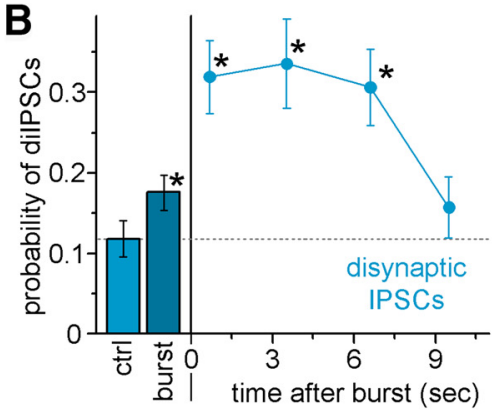

D

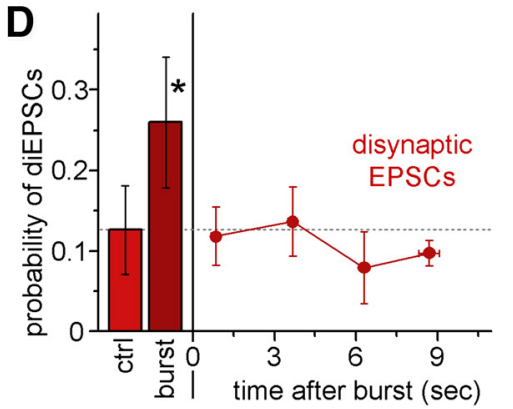

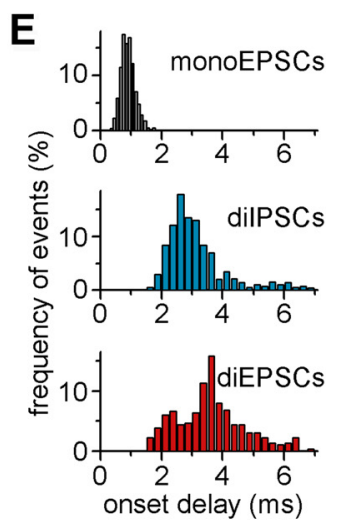

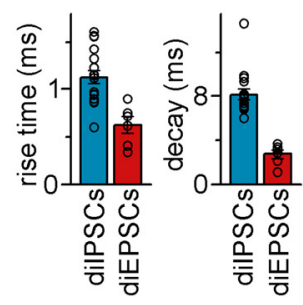

Figure 7. Differential effects of single bursts in individual MFs on dilPSCS and diEPSCS. $A$, Example traces: Synaptic events in a single randomly chosen CA3 pyramidal cell during the stimulation of a simultaneously recorded single C $33 \mathrm{GC}$ that was not monosynaptically connected to the pyramidal cell; the majority of the outward events are disynaptic GABAergic responses (dilPSCs; at - 50 $\mathrm{mV}$ using low chloride internal solution in postsynaptic cells), which presumably originate from one or more nonrecorded FF-IN(s); there is increased probability of dilPSC after single bursts in the MF (test). Black average traces represent the increased weight of disynaptic inhibition after the bursts. Right, Bar graphs represent summary data from dilPSC experiments. The bar graphs illustrate two types of dilPSC pairs: one in which the presynaptic MF source was a MF terminal (presumably originating from DG GCS), the other in which the MF source was a somatically recorded CA3 GC. There is a lack of differences in the burst-induced changes in the dilPSCs, suggesting that these two MF recording configurations induce similar burst-dependent effects on the CA3 feedforward inhibitory circuit. $\boldsymbol{B}$, Summary data indicating that the postburst time course of the increase in the probability of single MF-evoked dilPSCs. There is similarity of the time course to that of the burst-induced potentiation of the MF-EPSCs in FF-INs in Figure 1B. The increase in the probability of the dilPSCs during the burst (dark blue) was smaller than it was after the burst, consistent with the FF-IN data in Figure 2C. C, Example traces: diEPSC events recorded from a nonidentified fast-spiking interneuron during the activation of a CA3 GC; these diEPSCs presumably reflect the spiking of one or more nonrecorded pyramidal cell(s); there are frequent diEPSCs during the high-frequency bursts (inset), whereas the probability of diEPSCs remained unchanged after the bursts. $D$, Summary data: burst effects on the probability of diEPSCs evoked by MFs before, during (bars), and after bursts in the same individual MF (average data of 6 pairs, with 15 AP bursts at $150 \mathrm{~Hz}$ ); note the difference compared with the data in $\boldsymbol{B}$, and the similarity to the MF-EPSC pyramidal cell data in Figure $2 C .{ }^{*}$ mark significant difference in the probability of disynaptic events relative to control probabilities (before burst). $\boldsymbol{E}$, Distribution of the onset time delays of individual postsynaptic events measured from the time of the presynaptic AP, including monosynaptic EPSCs in postsynaptic FF-INs (gray), dilPSCs in CA3 pyramidal cells (blue), and diEPSCs in GABAergic cells (red). The 10\%-90\% rise time and decay time constants of the dilPSCs and diEPSCs are shown below. Each data point represents an individual pair.

corded FF-IN(s). In addition, these results were similar regardless of whether we evoked bursts in a giant MF terminal or in somatically recorded CA3 GCs (Szabadics et al., 2010) (Fig. 7A, bottom right inset, bar graphs; because DG GCs are much more numerous than CA3GCs, the MF terminal recordings must have overwhelmingly come from MFs of DG origin; therefore, these results also showed that the burst-related plasticity in FF-INs was similar with CA3 GC or DG GC inputs). In contrast, when we assessed the effect of single bursts in individual MFs on pyramidal cell activation by recording disynaptic EPSCs from CA3 FF-INs, we found a strong increase in the probability of diEPSCs during the bursts (control: $12.6 \pm 5.5 \%$, during burst: $26 \pm 8.1 \%, n=6$ pairs, $p=0.015, t_{(5)}=-3.649$, paired $t$ test), but no enhancement of the probability of diEPSCs after the bursts (Fig. 7C,D; probability of diIPSCs after test pulses 1.5-6.7 s following the burst: $10.6 \pm 2.9 \%, p=0.61, t_{(11)}=0.521$, paired $t$ test). These results supported our hypothesis that pyramidal cells were more sensitive to the bursts, whereas the inhibitory network preferentially represented the recent past of MF burst activity.

\section{Discussion}

\section{Unusual properties of postburst potentiation at MF-FF-} IN synapses

The results in this paper report the existence of a seconds-long, novel form of synaptic plasticity taking place between presynap- tic MFs and postsynaptic CA3 FF-INs. A major distinguishing feature of the plasticity is that it could be triggered by single, brief presynaptic burst activity consisting of as few as 3 APs at $150 \mathrm{~Hz}$, as well as by naturally occurring bursts recorded from GCs in vivo. The postburst potentiation also had an unusual time course, as it took almost a full second to develop, had no clear maximum, and persisted at a similar level for several seconds. An order of magnitude stronger stimulation paradigm (100 AP, delivered in $2.5 \mathrm{~s}$ ) applied to single GCs in hippocampal slice cultures have been shown to cause an increase in feedforward inhibition in the CA3 for $>10$ min (Mori et al., 2004, 2007). Apart from the distinct time course, the latter plasticity sharply differed from the postburst potentiation reported in this paper because it was expressed in GABAergic neurons as well as pyramidal cells (Mori et al., 2007). Similarly, the brief unitary burst-induced potentiation in interneurons reported in the current study also differs from the short-term facilitation and long-term MF plasticity taking place in CA3 pyramidal cells after cooperative stimulation of multiple GCs with spike trains consisting of tens of spikes associated with place field traversals (Gundlfinger et al., 2010). Therefore, our results reveal a novel form of synaptic plasticity evoked by physiologically relevant stimuli (Dobrunz and Stevens, 1999; Gundlfinger et al., 2010).

Interestingly, the cell-type specificity of the MF-induced postburst potentiation had two complementary aspects. On the one 
hand, it occurred similarly in both perisomatically (e.g., axoaxonic cells, $\mathrm{PV}+\mathrm{BCs}$ ) and dendritically projecting (e.g., ivy cells) FF-INs, despite their distinct intrinsic and synaptic properties, connectivity and local circuit functions (Ruediger et al., 2011; Somogyi et al., 2014). On the other hand, the postburst potentiation was much weaker or nonexistent in SLCs (which do not contribute to feedforward inhibition), highlighting the fact that the plasticity was cell type-specific, even among GABAergic cells. MFs are known to provide monosynaptic innervation to four distinct GABAergic cell types in the CA3, including cells with local (FF-INs, including PV cells, CCK+Ins, and ivy cells) and extrahippocampal (SLC) projections. SLCs are projection cells that mainly target the medial septum, and their primary dendrites are largely restricted to the stratum lucidum (and, to a lesser extent, the stratum pyramidale) (Gulyás et al., 1992; Jinno et al., 2007), with densely placed spines on the dendrites. A curious aspect of these cells is that, although they receive most of their inputs from MFs (Wittner et al., 2006), the existence of monosynaptic MF inputs is often revealed only after $>10$ APs at $50 \mathrm{~Hz}$, and our experiments showed that SLCs lacked MF postburst potentiation even under artificial conditions (high extracellular $\mathrm{Ca}^{2+}$ ) when the probability of successful EPSCs after presynaptic action potentials was increased. Interestingly, small MF terminals specialized to innervate GABA cells can be either filopodial extensions that emanate from the large MF terminals or en passant boutons. However, it is not known whether the FF-INs and SLCs are selectively innervated by specific small MF types, and it is also unclear whether the observed differences in the MF burstinduced potentiation between the FF-INs and SLCs correlates with the nature of the small MF presynaptic element.

In addition to the within-GABA cell group differences, the cell type specificity of the postburst plasticity was especially apparent when FF-INs and CA3 pyramidal cells were compared. Together, the results indicated a remarkable temporal differentiation of MF potentiation effects at the MF giant terminal to pyramidal cell versus the filopodial and en passant small bouton synapses on FF-INs (Acsády et al., 1998), with CA3 pyramidal cells displaying the well-known frequency facilitation during the bursts, whereas FF-INs exhibiting preferential augmentation of the MF responses for several seconds after the passage of a single brief MF burst. The significantly decreased delay and variability of onset of the MF-EPSCs during the postburst period in the FF-INs indicated accelerated, more precise, and more reliable release as being the key features of the postburst plasticity at MF-FF-IN synapses, perhaps through the involvement of enhanced vesicle priming (as suggested by the apparent convergence of the burst- and PDBu effects) (Rhee et al., 2002; Lou et al., 2008; Fioravante et al., 2014; Taschenberger et al., 2016). Curiously, various pharmacological manipulations of PKC, PKA, and mGluRs (Alle et al., 2001; Pelkey et al., 2005, 2008; Gundlfinger et al., 2010; Hainmüller et al., 2014) did not yield significant inhibition of the postburst potentiation in FF-INs, even in the presence of positive controls, raising the possible involvement of an unusual form of plasticity mechanism that will need to be investigated in the future.

In conclusion, different forms of short-term plasticity mechanisms have been identified as controllers of spike transfer at DG-CA3 connections (Nicoll and Schmitz, 2005; Evstratova and Tóth, 2014; Cherubini and Miles, 2015; Zucca et al., 2017) assisting in pattern completion and pattern separation tasks (Guzowski et al., 2004; Rolls and Treves, 2011). Local feedforward inhibitory circuits have been recognized to play key roles in controlling information transfer at MF-CA3 inputs, including short- term plasticity mechanisms at MF-FF-IN synapses lasting for tens of milliseconds (Szabadics and Soltesz, 2009; Torborg et al., 2010) and a $100-\mathrm{ms}$-long inhibition mediated by $\mathrm{GABA}_{\mathrm{B}}$ receptors in vivo (Zucca et al., 2017). Acting at considerably longer timescales, the potentiation of the MF inputs to FF-INs after a single, brief burst of APs in individual MFs reported in the present study was strong enough to cause a robust enhancement of disynaptic IPSCs, even in randomly sampled pyramidal cells that were not themselves monosynaptically connected to the activated individual MF. Therefore, it appears that single physiologically realistic bursts in individual GCs are able to increase feedforward inhibition in a sizeable portion of the CA3 network (e.g., at spatial scales corresponding to a hippocampal lamella partially represented in a hippocampal acute slice). Although recent results demonstrated that GCs typically possess only single place fields and fire at low frequencies (Danielson et al., 2017; GoodSmith et al., 2017; Senzai and Buzsáki, 2017), the sheer numerical abundance of GCs seems to imply that even the relatively sparse occurrence of burst discharges (Diamantaki et al., 2016) in individual GCs may collectively result in the CA3 FF-IN network being placed in a postburst potentiated state most of the time. Alternatively, the ability of solitary brief bursts in single GCs to powerfully enhance inhibition at the DG-CA3 interface in the seconds-long timescales of interburst intervals in vivo may play a role in setting the sensitivity of specific subsets of CA3 ensemble as a function of recent history of GC activity (e.g., during reentry of a recently visited place field where specific GCs fired burst of Aps) (Diamantaki et al., 2016), potentially regulating the contrast between representations of two similar events in CA3 circuits. Future tests of these not necessarily mutually exclusive hypotheses will help to develop a quantitative understanding of the ability of presynaptic GCs to recruit strong feedforward inhibition in populations of CA3 pyramidal cells, likely aided by in vivo experiments (PerníaAndrade and Jonas, 2014; Zucca et al., 2017) in combination with biologically realistic, data-driven large-scale computational network models (Bezaire et al., 2016). Although several aspects of the MF postburst potentiation in CA3 FF-INs will need to be explored further, the results reported in this paper are in general agreement with the notion that bursts represent a unique signal that is distinct from single spikes and even the arithmetically summed effects of single spikes (Lisman, 1997; Kaifosh and Losonczy, 2016).

\section{References}

Acsády L, Kamondi A, Sík A, Freund T, Buzsáki G (1998) GABAergic cells are the major postsynaptic targets of mossy fibers in the rat hippocampus. J Neurosci 18:3386-3403. Medline

Alle H, Jonas P, Geiger JR (2001) PTP and LTP at a hippocampal mossy fiber-interneuron synapse. Proc Natl Acad Sci U S A 98:14708-14713. CrossRef Medline

Apostolides PF, Milstein AD, Grienberger C, Bittner KC, Magee JC (2016) Axonal filtering allows reliable output during dendritic plateau-driven complex spiking in CA1 neurons. Neuron 89:770-783. CrossRef Medline

Augustin I, Rosenmund C, Südhof TC, Brose N (1999) Munc13-1 is essential for fusion competence of glutamatergic synaptic vesicles. Nature 400: 457-461. CrossRef Medline

Betz A, Ashery U, Rickmann M, Augustin I, Neher E, Südhof TC, Rettig J, Brose N (1998) Munc13-1 is a presynaptic phorbol ester receptor that enhances neurotransmitter release. Neuron 21:123-136. CrossRef Medline

Bezaire MJ, Raikov I, Burk K, Vyas D, Soltesz I (2016) Interneuronal mechanisms of hippocampal theta oscillations in a full-scale model of the rodent CA1 circuit. Elife 5:e18566. CrossRef Medline

Brunner J, Neubrandt M, Van-Weert S, Andrási T, Kleine Borgmann FB, Jessberger S, Szabadics J (2014) Adult-born granule cells mature through two functionally distinct states. Elife 3:e03104. CrossRef Medline 
Buzsáki G, Leung LW, Vanderwolf CH (1983) Cellular bases of hippocampal EEG in the behaving rat. Brain Res 287:139-171. Medline

Chamberland S, Evstratova A, Tóth K (2014) Interplay between synchronization of multivesicular release and recruitment of additional release sites support short-term facilitation at hippocampal mossy fiber to CA3 pyramidal cells synapses. J Neurosci 34:11032-11047. CrossRef Medline

Cherubini E, Miles R (2015) The CA3 region of the hippocampus: how is it? What is it for? How does it do it? Front Cell Neurosci 9:19. CrossRef Medline

Chu Y, Fioravante D, Leitges M, Regehr WG (2014) Calcium-dependent PKC isoforms have specialized roles in short-term synaptic plasticity. Neuron 82:859-871. CrossRef Medline

Danielson NB, Turi GF, Ladow M, Chavlis S, Petrantonakis PC, Poirazi P, Losonczy A (2017) In vivo imaging of dentate gyrus mossy cells in behaving mice. Neuron 93:552-559.e4. CrossRef Medline

Diamantaki M, Frey M, Berens P, Preston-Ferrer P, Burgalossi A (2016) Sparse activity of identified dentate granule cells during spatial exploration. Elife 5:e20252. CrossRef Medline

Dobrunz LE, Stevens CF (1999) Response of hippocampal synapses to natural stimulation patterns. Neuron 22:157-166. CrossRef Medline

Evstratova A, Tóth K (2014) Information processing and synaptic plasticity at hippocampal mossy fiber terminals. Front Cell Neurosci 8:28. CrossRef Medline

Fioravante D, Chu Y, Myoga MH, Leitges M, Regehr WG (2011) Calciumdependent isoforms of protein kinase $\mathrm{C}$ mediate posttetanic potentiation at the calyx of held. Neuron 70:1005-1019. CrossRef Medline

Fioravante D, Chu Y, de Jong AP, Leitges M, Kaeser PS, Regehr WG (2014) Protein kinase $\mathrm{C}$ is a calcium sensor for presynaptic short-term plasticity. Elife 3:e03011. CrossRef Medline

GoodSmith D, Chen X, Wang C, Kim SH, Song H, Burgalossi A, Christian KM, Knierim JJ (2017) Spatial representations of granule cells and mossy cells of the dentate gyrus. Neuron 93:677-690.e5. CrossRef Medline

Gulyás AI, Miettinen R, Jacobowitz DM, Freund TF (1992) Calretinin is present in non-pyramidal cells of the rat hippocampus: I. A new type of neuron specifically associated with the mossy fibre system. Neuroscience 48:1-27. CrossRef Medline

Gundlfinger A, Breustedt J, Sullivan D, Schmitz D (2010) Natural spike trains trigger short- and long-lasting dynamics at hippocampal mossy fiber synapses in rodents. PLoS One 5:e9961. CrossRef Medline

Guzowski JF, Knierim JJ, Moser EI (2004) Ensemble dynamics of hippocampal regions CA3 and CA1. Neuron 44:581-584. CrossRef Medline

Hainmüller T, Krieglstein K, Kulik A, Bartos M (2014) Joint CP-AMPA and group I mGlu receptor activation is required for synaptic plasticity in dentate gyrus fast-spiking interneurons. Proc Natl Acad Sci U S A 111: 13211-13216. CrossRef Medline

Harris KD, Hirase H, Leinekugel X, Henze DA, Buzsáki G (2001) Temporal interaction between single spikes and complex spike bursts in hippocampal pyramidal cells. Neuron 32:141-149. CrossRef Medline

Hashimotodani Y, Nasrallah K, Jensen KR, Chávez AE, Carrera D, Castillo PE (2017) LTP at hilar mossy cell-dentate granule cell synapses modulates dentate gyrus output by increasing excitation/inhibition balance. Neuron 95:928-943.e3. CrossRef Medline

Henze DA, Wittner L, Buzsáki G (2002) Single granule cells reliably discharge targets in the hippocampal CA3 network in vivo. Nat Neurosci 5:790-795. CrossRef Medline

Jinno S, Klausberger T, Marton LF, Dalezios Y, Roberts JD, Fuentealba P, Bushong EA, Henze D, Buzsáki G, Somogyi P (2007) Neuronal diversity in GABAergic long-range projections from the hippocampus. J Neurosci 27:8790-8804. CrossRef Medline

Jung MW, McNaughton BL (1993) Spatial selectivity of unit activity in the hippocampal granular layer. Hippocampus 3:165-182. CrossRef Medline

Junge HJ, Rhee JS, Jahn O, Varoqueaux F, Spiess J, Waxham MN, Rosenmund C, Brose N (2004) Calmodulin and Munc13 form a Ca ${ }^{2+}$ sensor/effector complex that controls short-term synaptic plasticity. Cell 118:389401. CrossRef Medline

Kaifosh P, Losonczy A (2016) Mnemonic functions for nonlinear dendritic integration in hippocampal pyramidal circuits. Neuron 90:622-634. CrossRef Medline

Korogod N, Lou X, Schneggenburger R (2007) Posttetanic potentiation critically depends on an enhanced $\mathrm{Ca}(2+)$ sensitivity of vesicle fusion mediated by presynaptic PKC. Proc Natl Acad Sci U S A 104:1592315928. CrossRef Medline

Lawrence JJ, McBain CJ (2003) Interneuron diversity series: containing the detonation-feedforward inhibition in the CA3 hippocampus. Trends Neurosci 26:631-640. CrossRef Medline

Lisman JE (1997) Bursts as a unit of neural information: making unreliable synapses reliable. Trends Neurosci 20:38-43. CrossRef Medline

Lorteije JA, Rusu SI, Kushmerick C, Borst JG (2009) Reliability and precision of the mouse calyx of held synapse. J Neurosci 29:13770-13784. CrossRef Medline

Lou X, Korogod N, Brose N, Schneggenburger R (2008) Phorbol esters modulate spontaneous and $\mathrm{Ca}^{2+}$-evoked transmitter release via acting on both Munc13 and protein kinase C. J Neurosci 28:8257-8267. CrossRef Medline

Mori M, Abegg MH, Gähwiler BH, Gerber U (2004) A frequencydependent switch from inhibition to excitation in a hippocampal unitary circuit. Nature 431:453-456. CrossRef Medline

Mori M, Gähwiler BH, Gerber U (2007) Recruitment of an inhibitory hippocampal network after bursting in a single granule cell. Proc Natl Acad Sci U S A 104:7640-7645. CrossRef Medline

Neubrandt M, Oláh VJ, Brunner J, Szabadics J (2017) Feedforward inhibition is randomly wired from individual granule cells onto CA3 pyramidal cells. Hippocampus 27:1034-1039. CrossRef Medline

Nicoll RA, Schmitz D (2005) Synaptic plasticity at hippocampal mossy fibre synapses. Nat Rev Neurosci 6:863-876. CrossRef Medline

Pelkey KA, Lavezzari G, Racca C, Roche KW, McBain CJ (2005) mGluR7 is a metaplastic switch controlling bidirectional plasticity of feedforward inhibition. Neuron 46:89-102. CrossRef Medline

Pelkey KA, Topolnik L, Yuan XQ, Lacaille JC, McBain CJ (2008) Statedependent cAMP sensitivity of presynaptic function underlies metaplasticity in a hippocampal feedforward inhibitory circuit. Neuron 60:980-987. CrossRef Medline

Pernía-Andrade AJ, Jonas P (2014) Theta-gamma-modulated synaptic currents in hippocampal granule cells in vivo define a mechanism for network oscillations. Neuron 81:140-152. CrossRef Medline

Rancz EA, Ishikawa T, Duguid I, Chadderton P, Mahon S, Häusser M (2007) High-fidelity transmission of sensory information by single cerebellar mossy fibre boutons. Nature 450:1245-1248. CrossRef Medline

Rhee JS, Betz A, Pyott S, Reim K, Varoqueaux F, Augustin I, Hesse D, Südhof TC, Takahashi M, Rosenmund C, Brose N (2002) Beta phorbol esterand diacylglycerol-induced augmentation of transmitter release is mediated by Munc13s and not by PKCs. Cell 108:121-133. CrossRef Medline

Rolls ET, Treves A (2011) The neuronal encoding of information in the brain. Prog Neurobiol 95:448-490. CrossRef Medline

Rosenmund C, Sigler A, Augustin I, Reim K, Brose N, Rhee JS (2002) Differential control of vesicle priming and short-term plasticity by Munc13 isoforms. Neuron 33:411-424. CrossRef Medline

Ruediger S, Vittori C, Bednarek E, Genoud C, Strata P, Sacchetti B, Caroni P (2011) Learning-related feedforward inhibitory connectivity growth required for memory precision. Nature 473:514-518. CrossRef Medline

Salin PA, Scanziani M, Malenka RC, Nicoll RA (1996) Distinct short-term plasticity at two excitatory synapses in the hippocampus. Proc Natl Acad Sci U S A 93:13304-13309. CrossRef Medline

Senzai Y, Buzsáki G (2017) Physiological properties and behavioral correlates of hippocampal granule cells and mossy cells. Neuron 93:691704.e5. CrossRef Medline

Shigemoto R, Kinoshita A, Wada E, Nomura S, Ohishi H, Takada M, Flor PJ, Neki A, Abe T, Nakanishi S, Mizuno N (1997) Differential presynaptic localization of metabotropic glutamate receptor subtypes in the rat hippocampus. J Neurosci 17:7503-7522. Medline

Somogyi P, Katona L, Klausberger T, Lasztóczi B, Viney TJ (2014) Temporal redistribution of inhibition over neuronal subcellular domains underlies state-dependent rhythmic change of excitability in the hippocampus. Philos Trans R Soc Lond B Biol Sci 369:20120518. CrossRef Medline

Spruston N, Lübke J, Frotscher M (1997) Interneurons in the stratum lucidum of the rat hippocampus: an anatomical and electrophysiological characterization. J Comp Neurol 385:427-440. CrossRef Medline

Szabadics J, Soltesz I (2009) Functional specificity of mossy fiber innervation of GABAergic cells in the hippocampus. J Neurosci 29:4239-4251. CrossRef Medline

Szabadics J, Varga C, Brunner J, Chen K, Soltesz I (2010) Granule cells in the CA3 area. J Neurosci 30:8296-8307. CrossRef Medline 
Taschenberger H, Woehler A, Neher E (2016) Superpriming of synaptic vesicles as a common basis for intersynapse variability and modulation of synaptic strength. Proc Natl Acad Sci U S A 113:E4548-E4557. CrossRef Medline

Torborg CL, Nakashiba T, Tonegawa S, McBain CJ (2010) Control of CA3 output by feedforward inhibition despite developmental changes in the excitationinhibition balance. J Neurosci 30:15628-15637. CrossRef Medline

Tóth K, McBain CJ (1998) Afferent-specific innervation of two distinct AMPA receptor subtypes on single hippocampal interneurons. Nat Neurosci 1:572-578. CrossRef Medline

Tóth K, Suares G, Lawrence JJ, Philips-Tansey E, McBain CJ (2000) Differential mechanisms of transmission at three types of mossy fiber synapse. J Neurosci 20:8279-8289. Medline

Viney TJ, Lasztoczi B, Katona L, Crump MG, Tukker JJ, Klausberger T, Somogyi P (2013) Network state-dependent inhibition of identified hippocampal CA3 axo-axonic cells in vivo. Nat Neurosci 16:1802-1811. CrossRef Medline

Vyleta NP, Jonas P (2014) Loose coupling between $\mathrm{Ca}^{2+}$ channels and release sensors at a plastic hippocampal synapse. Science 343:665-670. CrossRef Medline
Vyleta NP, Borges-Merjane C, Jonas P (2016) Plasticity-dependent, full detonation at hippocampal mossy fiber-CA3 pyramidal neuron synapses. Elife 5:e17977. CrossRef Medline

Wierda KD, Toonen RF, de Wit H, Brussaard AB, Verhage M (2007) Interdependence of PKC-dependent and PKC-independent pathways for presynaptic plasticity. Neuron 54:275-290. CrossRef Medline

Wittner L, Henze DA, Záborszky L, Buzsáki G (2006) Hippocampal CA3 pyramidal cells selectively innervate aspiny interneurons. Eur J Neurosci 24:1286-1298. CrossRef Medline

Wu XS, Wu LG (2001) Protein kinase c increases the apparent affinity of the release machinery to $\mathrm{Ca}^{2+}$ by enhancing the release machinery downstream of the $\mathrm{Ca}^{2+}$ sensor. J Neurosci 21:7928-7936. Medline

Xu W, Morishita W, Buckmaster PS, Pang ZP, Malenka RC, Südhof TC (2012) Distinct neuronal coding schemes in memory revealed by selective erasure of fast synchronous synaptic transmission. Neuron 73:9901001. CrossRef Medline

Zucca S, Griguoli M, Malézieux M, Grosjean N, Carta M, Mulle C (2017) Control of spike transfer at hippocampal mossy fiber synapses in vivo by GABAA and GABAB receptor-mediated inhibition. J Neurosci 37:587598. CrossRef Medline 